\title{
Inner Ear Genes Underwent Positive Selection and Adaptation in the Mammalian Lineage
}

\author{
Francisco Pisciottano, ${ }^{\dagger, 1,2}$ Alejandro R. Cinalli, ${ }^{\dagger, 1,2}$ Juan Matías Stopiello, ${ }^{\dagger, 1,2}$ Valeria C. Castagna, ${ }^{3}$ \\ Ana Belén Elgoyhen, ${ }^{1,2}$ Marcelo Rubinstein, ${ }^{1,2,4}$ María Eugenia Gómez-Casati, ${ }^{1,2,3}$ and \\ Lucía F. Franchini ${ }^{* 1,2}$ \\ ${ }^{1}$ Instituto de Investigaciones en Ingeniería Genética y Biología Molecular (INGEBI), Buenos Aires, Argentina \\ ${ }^{2}$ Consejo Nacional de Investigaciones Científicas y Técnicas (CONICET), Buenos Aires, Argentina \\ ${ }^{3}$ Instituto de Farmacología, Facultad de Medicina, Universidad de Buenos Aires, Buenos Aires,Argentina \\ ${ }^{4}$ Facultad de Ciencias Exactas y Naturales (FCEN), Universidad de Buenos Aires, Buenos Aires,Argentina \\ ${ }^{\dagger}$ These authors contributed equally to this work. \\ *Corresponding author: E-mail: franchini@dna.uba.ar. \\ Associate editor: Emma Teeling
}

\begin{abstract}
The mammalian inner ear possesses functional and morphological innovations that contribute to its unique hearing capacities. The genetic bases underlying the evolution of this mammalian landmark are poorly understood. We propose that the emergence of morphological and functional innovations in the mammalian inner ear could have been driven by adaptive molecular evolution.

In this work, we performed a meta-analysis of available inner ear gene expression data sets in order to identify genes that show signatures of adaptive evolution in the mammalian lineage. We analyzed $\sim 1,300$ inner ear expressed genes and found that $13 \%$ show signatures of positive selection in the mammalian lineage. Several of these genes are known to play an important function in the inner ear. In addition, we identified that a significant proportion of genes showing signatures of adaptive evolution in mammals have not been previously reported to participate in inner ear development and/or physiology. We focused our analysis in two of these genes: STRIP2 and ABLIM2 by generating null mutant mice and analyzed their auditory function. We found that mice lacking Strip2 displayed a decrease in neural response amplitudes. In addition, we observed a reduction in the number of afferent synapses, suggesting a potential cochlear neuropathy. Thus, this study shows the usefulness of pursuing a high-throughput evolutionary approach followed by functional studies to track down genes that are important for inner ear function. Moreover, this approach sheds light on the genetic bases underlying the evolution of the mammalian inner ear.
\end{abstract}

Key words: inner ear, evolution, STRIP2, ABLIM2, prestin, synemin, spectrin.

\section{Introduction}

The auditory system of mammals is distinguished by a middle ear composed of three ossicles and an inner ear, with an auditory organ of Corti that possesses two types of specialized sensory hair cells (HCs), inner (IHCs) and outer hair cells (OHCs) (Manley 2000, 2012; Fritzsch et al. 2013; Elliott et al. 2018). The organ of Corti seems to have appeared early on in the evolution of therapsids (reptile ancestors of mammals) and it is derived from the basilar papilla of amniotes (Manley 2000, 2010; Fritzsch et al. 2013). In monotremes or egg-laying mammals (prototheria), the organ of Corti possess IHCs and $\mathrm{OHCs}$ but they are organized in several rows (Ladhams and Pickles 1996), whereas there is only one row of IHCs and three rows of OHCs in therian mammals (Slepecky 1996), indicating further specialization in marsupials and placentals. Furthermore, the elongation of the organ of Corti contained in the cochlear duct in therian mammals (Schultz et al. 2017) allowed the acquisition of unique hearing capacities among vertebrates, including high-frequency sensitivity developed to an extreme level in some groups such as bats and whales (Manley 2012; Fritzsch et al. 2013). In the mammalian cochlea, IHCs and OHCs display a clear division of labor: IHCs receive and relay sound information behaving as the true sensory cells, whereas OHCs amplify sound information. Thus, IHCs which are the primary transducers, release glutamate to excite the sensory fibers of the cochlear nerve and OHCs act as biological motors to amplify the motion of the sensory epithelium. OHCs endowed mammals with a novel cochlear amplification mechanism known as somatic electromotility, crucial for auditory sensitivity and frequency selectivity (Brownell et al. 1985; Ashmore 1987; Dallos 2008; Ashmore et al. 2010). In addition, these functional rearrangements at the inner ear level were accompanied by evolutionary changes at the auditory pathway in the central nervous system which underwent massive rearrangement in the tetrapod lineage (Grothe et al. 2004).

The genetic remodeling that enabled the acquisition of morphological and functional novelties in the mammalian 
inner ear is poorly understood. Through mutant mice analysis, it has been shown that several transcription factors (TFs) are key to the development of the entire organ of Corti such as N-Myc, Pax2, and Gata3 (Bouchard et al. 2010; Kopecky et al. 2012; Duncan and Fritzsch 2013). However, molecular evolutionary studies linking the evolution of these key developmental genes to the evolution of the mammalian inner ear are still missing. In this regard, recent findings have shown that several key inner ear genes underwent molecular adaptations that are probably linked to the evolution of the highfrequency hearing in mammals (Franchini and Elgoyhen 2006; Okoruwa et al. 2008; Elgoyhen and Franchini 2011). For example, SLC26A5 (encoding prestin), one of the first inner ear genes where signatures of positive selection in mammals were found (Franchini and Elgoyhen 2006), is a protein of the solute carrier (SLC) family directly involved in active amplification mechanisms of the mammalian cochlea (Belyantseva et al. 2000; Zheng et al. 2000). Phylogenetic analyses of prestin and other SLC proteins have uncovered significant adaptive changes in the prestin amino-acid sequence, which have occurred essentially after the split between the avian and mammalian lineages. These adaptive changes have been related to the emergence of electromotility in mammals (Franchini and Elgoyhen 2006; Okoruwa et al. 2008; Elgoyhen and Franchini 2011). In addition, we have recently shown that adaptive mutations of spectrin- $\beta \vee$ occurred in the mammalian, but not the avian lineage, and were accompanied by substantial changes in the protein distribution within inner ear HCs (Cortese et al. 2017).

The deep evolutionary rearrangements that occurred in the mammalian inner ear involved the appearance of new cellular systems and novel functions, which probably required evolutionary changes in many proteins. In this work, we aimed to identify the genetic bases underlying the evolution of the inner ear in mammals. To identify inner ear genes (i.e., genes that play a functional role in the inner ear) that adaptively evolved in the mammalian lineage, we first analyzed several expression databases and searched for signatures of positive selection by comparing synonymous and nonsynonymous substitution rates in gene coding sequences. We found 165 genes expressed in different cell types of the inner ear displaying signatures of positive selection in the mammalian lineage. Then, for a selected group of these positively selected genes, we analyzed in detail their expression pattern in the inner ear. Using this information, we chose two genes with unknown function in the inner ear: STRIP2 (from Striatin Interacting Protein 2) and ABLIM2 (Actin Binding LIM domain 2) which were functionally characterized by generating novel strains of mutant mice by CRISPR/Cas9 technology. This work sheds light on the genetic basis underlying the evolution of the mammalian inner ear and highlights the usefulness of evolutionary studies to pinpoint key functional genes.

\section{Results}

HCs Transcriptome Evolutionary Analyses

Several groups have recently sought to obtain the transcriptomes of HCs and other cell types in the cochlea and vestibular sensory epithelia, in attempts to reveal the genetic basis underlying the molecular mechanisms of hearing and balance. Here, we analyzed three mouse inner ear expression data sets generated using different methodologies (Liu et al. 2014; Burns et al. 2015; Scheffer et al. 2015) in order to identify those genes differentially expressed in different cellular types of the inner ear (e.g., IHCs, OHCs, and supporting cells) and that most probably underlie their distinctive functional properties. To that end we developed a high-throughput analysis pipeline that included an automatized method to retrieve and align the sequences of seven selected species representing all vertebrate classes and then run a branch-site evolutionary test (fig. 1a and supplementary fig. S1a, Supplementary Material online). From these data sets, we selected fulllength protein-coding genes represented by only one ortho$\log$ in each included species.

We first analyzed transcripts differentially expressed in either IHCs or OHCs manually dissected from adult mouse cochleae (Liu et al. 2014). In this work, gene expression profiles were determined by hybridizing total RNA with a GeneChip microarray (GeneChip Mouse Gene 2.0 ST Arrays). The authors found a total of 16,117 transcripts being expressed in both populations of HCs, whereas they found 1,296 genes being significantly differentially expressed in IHCs and 198 being significantly differentially expressed in OHCs (supplementary table S1, Supplementary Material online). After filtering out the 1,193 reported IHC transcripts according to the aforementioned inclusion criteria we found 477 that met our standards but two of them showed incomplete sequence information. Thus, we analyzed 475 full-length coding transcripts by the branch-site method and detected 37 genes (7.8\%) with signatures of positive selection in the mammalian lineage (table 1). Then, we analyzed the reported 198 OHCs differentially expressed transcripts, of which only 88 fulfilled the inclusion criteria. The branch-site positive selection analysis for these $88 \mathrm{OHCs}$ genes consistently retrieved 11 (12.5\%) positively selected genes at $P<0.05$ (table 1 ). Forty genes from these 198 OHCs transcripts were automatically excluded from our high-throughput pipeline due to one-tomany orthology relationships among the 7 selected species and 64 presented incomplete sequence information in one or more of the analyzed species (supplementary table S2, Supplementary Material online). However, we decided to study the 40 genes with complex orthology by unfolding the analysis in as many runs as necessary and found 4 additional positively selected genes (supplementary table S3, Supplementary Material online).

The second data set we analyzed used fluorescence-activated cell sorter (FACS) isolation of HCs from mice at three postnatal stages ( $\mathrm{P0}, \mathrm{P} 4$, and $\mathrm{P} 7$ ) and one embryonic stage (E16), which were then subjected to an RNA-Seq protocol (Scheffer et al. 2015). FACS isolation allowed the authors to separate between supporting and HCs thanks to the expression of GFP in HCs. From the 18,199 genes that they found being expressed in mouse inner ear at the different stages analyzed, the authors discriminated genes differentially expressed in hair or surrounding cells from the cochlea and the utricle using expression values and setting a fold-change 

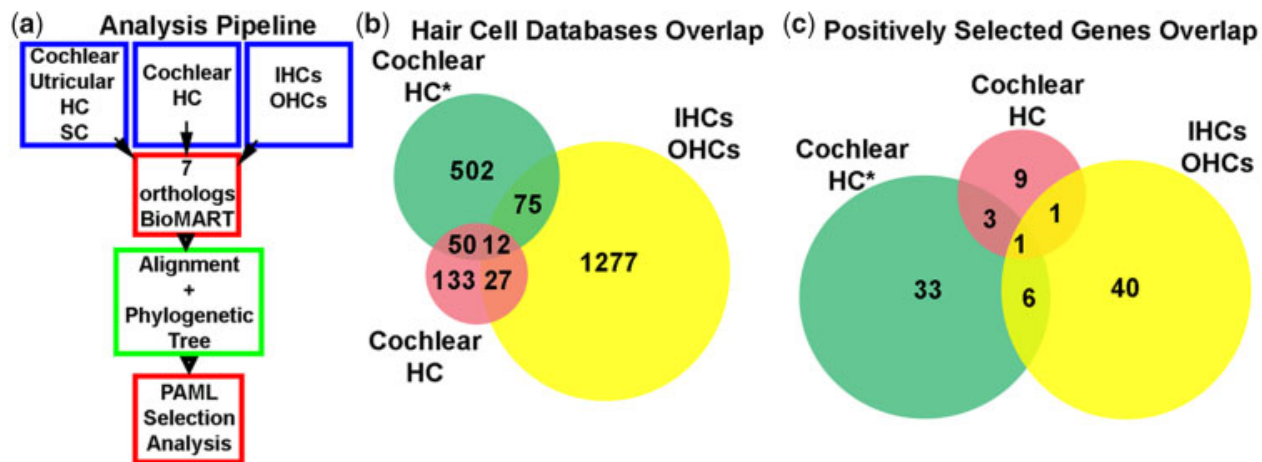

FIG. 1. Analysis pipeline and different expression databases overlap. (a) An schematic diagram of the evolutionary analysis pipeline. (b) Gene overlap among the three cochlear HC expression data sets analyzed. (c) Overlap among positively selected cochlear genes identified in the different databases. The only gene that is shared among the positively selected genes identified from the three databases is STRIP2.

Table 1. Positive Selection Analysis on Differentially Expressed IHCs and OHCs Genes.

\begin{tabular}{|c|c|c|}
\hline $\begin{array}{l}\text { Total Genes/ } \\
\text { One-to-One } \\
\text { Genes/Analyzed } \\
\text { Genes }\end{array}$ & Pos. Sel. Genes & Pos. Sel. Gene Symbols \\
\hline \multicolumn{3}{|c|}{ Inner hair cells differentially expressed genes } \\
\hline $1193 / 477 / 475^{\mathrm{a}}$ & $37(7.8 \%)$ & $\begin{array}{l}\text { ADGRG6, AKAP12, BOD1L1, } \\
\text { CTSA, DFNB31, DMXL2, } \\
\text { EDEM2, FAM65C, FASN, FAT4, } \\
\text { FLAD1, GALK1, GOLGA1, } \\
\text { GRAMD1C, IDH1, IFT46, } \\
\text { IFT74, IRAK4, LARP1, MRPL3, } \\
\text { NRCAM, PEAK1, PPP1R36, } \\
\text { PREPL, PROM2, QSER1, } \\
\text { RHPN2, ROCK1, SLU7, SQLE, } \\
\text { SSFA2, TJAP1, TMEM11, } \\
\text { USP6NL, UTP15, WDR36, } \\
\text { ZNF318 }\end{array}$ \\
\hline \multicolumn{3}{|c|}{ Outer hair cells differentially expressed genes } \\
\hline $198 / 88 / 88$ & $11(12.5 \%)$ & $\begin{array}{l}\text { ATP13A1, GCN1L1, CLU, STRIP2, } \\
\text { ATP8B1, SYNM, LMOD3, } \\
\text { ABLIM2, SPTB, SLC26A5, } \\
\text { ZNF516 }\end{array}$ \\
\hline
\end{tabular}

Note.-Data obtained from Liu et al. (2014) (see also supplementary table S1, Supplementary Material online). Positive selected genes reported are consistent among three runs.

${ }^{a}$ Two one-to-one IHC differentially expressed genes (UNC80 and NKAIN2) had to be excluded due to partial sequences and complete deletions option, which rendered an alignment that could not be processed.

threshold (see supplementary table S1, Supplementary Material online, for more detail). Among these demarcated clusters of genes, we decided to study those that include genes differentially expressed in postnatal cochlear HCs, utricular HCs and surrounding or supporting cells. From 222 postnatal cochlear HCs enriched transcripts, 82 met our analysis criteria and 14 (17.1\%) showed to be under adaptive evolution in mammals (table 2). From 41 differentially expressed postnatal utricular $\mathrm{HC}$ genes, 16 met our analysis criteria and we identified 4 (25\%) showing signatures of positive selection in the basal mammalian tree branch (table 2). In addition, from 1,071 genes differentially expressed in inner ear surrounding or supporting cells, 390 could be analyzed and we spotted 53 genes (13.6\%; table 2 ) with signatures of positive
Table 2. Positive Selection Analysis on Genes Enriched in Hair and Surrounding Cells from Cochlear and Utricular Samples in Postnatal Mice.

Total Genes/ Pos. Sel. Genes Pos. Sel. Gene Symbols
One-to-One
Genes/Analyzed
Genes

Cochlear hair cells enriched genes

$$
\text { 222/82/82 } 14(17.1 \%)
$$

ZNF750, CTIF, PHGDH, PARD6A, SIX2, STRIP2, SMOC2, TMPRSS7, MYOM1, MYO3A, TJP3, KIAA1467, ZNF503, SLC26A5

Utricular hair cells enriched genes $41 / 16 / 16 \quad 4(25.0 \%)$ C5ORF49, CCDC108, SLC15A5,
DNAH5

Supporting cells enriched genes $1,071 / 390 / 390 \quad 53(13.6 \%)$

DCT, NOD1, SEMA3B, CSPG4, SOD3, EGFLAM, COL16A1, COL5A1, CPM, SH3PXD2B, LIPA, CTSE, COL15A1, VCAM1, CD74, FGFR4, CD44, ATP10B, KCNK5, HIC1, PLXND1, PRSS12, PDGFRB, GPR65, MYBPC1, SLCO1A2, ENPP1, AFAP1L2, LCAT, COL4A1, CACNA1E, SVEP1, COL4A5, FAT4, HEXB, TMEM45A, ENPEP, COL9A3, IGSF10, ACSF2, SERPING1, PEAR1, CGNL1, SLC26A11, STEAP3, TIE1, ITGAV, ACADL, LAMB2, ARAP3, P3H3, ADGRA2, CILP2

Hair cells genes not enriched in cochlea or utricle

\begin{tabular}{ll}
$432 / 154 / 154$ & ATP8B1, DLK2, DISP2, \\
& TMEM184A, PKD2L1, SPAG6, \\
& MYOT, DRC3, DUSP27, DGKG, \\
& ARMC3, PADI2, WWC1, \\
& WDR78, LRRC6, EFHC2, \\
& ABLIM2, GRAMD1C, CDH15, \\
& HSPA4L, CHRNG, TTC34, \\
& SLC7A4, DRC7, NEFL \\
\hline
\end{tabular}

Note.-Data obtained from Scheffer et al. (2015). Positive selected genes reported are consistent among three runs.

selection in mammals. In addition, we analyzed a group of 432 genes that are expressed in HCs but did not show differential expression compared with supporting cells. We were able to 
retrieve 154 that follow our analysis criteria and we found that 25 genes (16.2\%; table 2) displayed signatures of positive selection.

The third data set analyzed was obtained by applying single-cell RNA-Seq transcriptomics to newborn mice (P1) inner ear cells (Burns et al. 2015). Single cells were procured using microfluidics-based protocols. Separation of the different cells types of the cochlea and the utricle was attained through the expression of different specific cell markers expressed by the transgenic mouse lines used to obtain the cells (supplementary table S1, Supplementary Material online). From this work, we analyzed a set of 639 cochlear $\mathrm{HC}$-specific genes and we identified in this data set a total of 248 protein-coding genes that met our analysis criteria, however 3 of these genes had to be excluded from the pipeline because of incomplete sequence information and then 245 genes were analyzed. Triplicate branch-site analysis consistently rendered the same $43(17.5 \%)$ positive selected genes $(P<0.05$; table 3$)$.

In general, most of the genes that could not be analyzed by our approach were noncoding genes or presented incomplete orthology sequence information (one or more ortholog sequences were absent in genomic databases) or multiple orthology in one or more species (supplementary table S2, Supplementary Material online). The impossibility to analyze the evolution of these genes suggests that the number of genes that underwent positive selection in the mammalian lineage is possibly underestimated.

Since the databases we analyzed were mainly assembled using expression in postnatal stages and in order to better assess the evolutionary history of the mammalian inner ear, we also analyzed a manually collected data set of TFs that have been reported to be key in inner ear development and that are expressed very early on during development. Thus, we analyzed $23 \mathrm{TF}$ and diffusible factors and found that most of them are extremely well conserved across vertebrates, suggesting that the evolution of their coding sequences did not play a significant role in mammalian inner ear changes. Only the gene helix-loop-helix $1(\mathrm{NHLH} 1)$ exhibited signatures of positive selection in the mammalian lineage (supplementary table S3, Supplementary Material online). It is interesting to note that it has been reported that this gene it is expressed very early on in the developing organ of Corti but its function in the development of the inner ear has not been completely established (Kruger et al. 2006).

\section{Overlap among Different Expression Databases}

When comparing the three cochlear HCs data sets, we found that only 12 transcripts were present in all databases (fig. 1b). Even though the three studies aimed to obtain the HC transcriptome, the low level of overlap in the three data sets could be possibly explained by the different methodologies applied and also due to the different developmental stages included (perinatal/juvenile versus adult). Although Scheffer et al. (2015) isolated genes preferentially expressed in newborn cochlear or utricular $\mathrm{HC}$ over those expressed in surrounding cells, Liu et al. (2014) reported OHC genes differentially expressed in $\mathrm{OHCs}$ in comparison to $\mathrm{IHCs}$, and vice versa in
Table 3. Positive Selection Analysis on Cochlear Hair Cells Genes of Newborn Mice Inner Ears.

Total Genes/ Pos. Sel. Genes Pos. Selected Gene Symbols
One-to-One
Genes/Analyzed
Genes

Cochlear hair cells specific genes

$639 / 248 / 245^{\mathrm{a}} \quad 43(17.5 \%)$

CCDC189, TPRN, IQUB, DUSP27, LRP2, RHPN2, ABCC1, CFAP65, WDR78, GNMT, AP3B2, LAMC2, C9orf114, PGAP1, CDH23, PTPN6, DRC3, PEAK1, DGKG, ZZEF1, STRIP2, EFHC1, ADGRV1, SMOC2, DISP2, TMPRSS7, TJAP1, KCNS3, TJP3, GAS6, LMOD3, ABLIM2, OBSCN, MAP2, MMS19, DZIP1L, DMXL2, TMEM184A, CHRNA10, DLK2, SLC7A4, C2CD2L, KIF9

NotE.-Data obtained from Burns et al. (2015). Positive selected genes reported are consistent among three runs.

${ }^{\mathrm{a}}$ Three one-to-one $\mathrm{HC}$ differentially expressed genes (RHBDF1, KNDC1, and GTF3C5) had to be excluded due to partial sequences and complete deletions option, which rendered an alignment that could not be processed.

adult mice. Similar to Scheffer et al., Burns et al. (2015) reported genes differentially expressed in $\mathrm{HC}$ in comparison to supporting cells, but only 62 genes were shared by these two data sets even though both studies analyzed perinatal stages including P0 (fig. 1b). This low level of overlap suggests that the study of the three data sets ensured a more comprehensive analysis and includes a higher number of transcripts, increasing the chances of detecting more inner ear genes under positive selection. In addition, this also suggests that as more data sets become available including more developmental stages and using different methodologies more genes under selection will be possibly found.

Regarding the positive selected genes identified, only STRIP2 was present in the three databases (fig. 1c). In addition, among 14 postnatal HC (Scheffer et al. 2015) and $48 \mathrm{IHC}$ or $\mathrm{OHC}$ (11 from OHCs and 37 from IHCs) (Liu et al. 2014) positively selected genes, we found only 2 genes present in both data sets: SLC26A5 and STRIP2 (fig. 1c). On the other hand, between the 43 and 48 positively selected genes identified in the Burns et al. (2015) and in the Liu et al (2014) data sets, we found that 7 overlapped, STRIP2, ABLIM2, Leimodin 3 (LMOD3), DMX like 2 (DMXL2), Rhophilin Rho GTPase Binding Protein 2 (RHPN2), Pseudopodium Enriched Atypical Kinase 1 (PEAK1), and Tight Junction Associated Protein 1 (TJAP1) (fig. 1c). Altogether, these data indicated that despite the source of the transcriptome and the small overlap among the databases a few genes are consistently present and identified as being under positive selection by our evolutionary analysis.

Functional Enrichment Analysis of OHCs and IHCs Differentially Expressed Genes

In order to analyze if the OHCs and IHCs positively selected genes shared associated functions, we carried out a functional 
enrichment analysis using the DAVID tool for functional annotation (https://david.ncifcrf.gov/) (Huang da et al. 2009). The results indicated that the most enriched functional term for the category of molecular function in the 11 $\mathrm{OHCs}$ positively selected genes was "cytoskeletal protein binding" ([Gene ontology (GO): 0008092] [P=3.02e-5; supplementary table $\mathrm{S} 5$, Supplementary Material online]). Moreover, the second most enriched term of this category was "structural constituent of the cytoskeleton" ([GO: 0005200 $] \quad[P=1.5 \mathrm{e}-2$; supplementary table $S 5$, Supplementary Material online]). The remaining seven enriched terms reported in the results were associated with transport through the membrane of ions and other molecules (supplementary table S5, Supplementary Material online). The enrichment in cytoskeleton functional proteins found in the $11 \mathrm{OHCs}$ positive selected genes is specific for this particular gene subset and not product of an inherited overrepresentation of proteins related to cytoskeleton in the list of the $88 \mathrm{OHCs}$, since the GO terms that better apply to the genes in this data set are from completely different functional categories (supplementary table S6, Supplementary Material online). We also performed a functional enrichment of the 11 positively selected genes, but this time using the list of $88 \mathrm{OHCs}$ genes analyzed, as background. This analysis returned a single functional enrichment term from these two lists, "cytoskeleton binding protein" (GO: 0008092) (2.2e-3), thus confirming the previous result. A functional enrichment analysis was also performed for the 37 positively selected genes identified in the IHC differentially expressed genes data set (Liu et al. 2014) and it did not yield any enriched functional GO term.

Altogether, these findings indicate that the OHCs genes that underwent positive selection could have contributed to the acquisition of the highly specialized cytoskeleton present in these cells that underlies its distinctive functional properties, including somatic electromotility.

\section{Extended Evolutionary Analyses of OHCs Positively Selected Genes}

Because of the importance of OHCs in mammalian audition, and to confirm the results of our exploratory analysis, we increased the statistical power of the evolutionary analysis by including more vertebrate species for all OHCs differentially expressed genes displaying signatures of adaptive evolution (table 1). For this analysis, we used all available sequences of vertebrate species and confirmed the results previously obtained (table 4), demonstrating that our approach using seven representative vertebrate species is sufficiently robust to identify genes with strong signals of adaptive evolution.

The inclusion of more species allowed us to detect codon sites with signatures of positive selection (table 4). We also used PROVEAN (Choi et al. 2012) to predict the potential functional impact of positively selected sites in the mammalian lineage for the deeply analyzed OHCs differentially expressed genes. We found (supplementary table S7, Supplementary Material online) that the majority of the genes ( 8 out of 11) displayed 1 or more evolutionary driven amino acid changes that were predicted to have a functional effect.
On the other hand, we tested the lineage leading to birds and found no evidence of positive selection for most genes except for synemin (SYNM) and clusterin (CLU) genes (table 4 and supplementary table S8, Supplementary Material online). Since HCs present in bird basilar papilla apparently remained in a more primitive state (Manley et al. 2004; Elliott et al. 2018) probably underwent less evolutionary changes or maybe the evolution of their particular phenotypical features involved different genes and pathways. More studies will be necessary to test this hypothesis.

We also tested the evolution of these OHCs genes in the tetrapod lineage and found extensive signatures of positive selection, suggesting that remodeling of these genes happened in several moments of the vertebrate history (supplementary table S6, Supplementary Material online). Besides, in order to better assess the evolution of this lineage, we analyzed the tetrapod lineage including the coelacanth Latimeria menadoensis sequence, a lobed-finned fish that possesses a tetrapodlike ear but that lives in an aquatic environment (Fritzsch 1987; Bernstein 2003). We found that in most genes the signature of positive selection is maintained when including this sequence (supplementary table S6, Supplementary Material online).

In order to test if the mammalian lineage underwent divergent selection, we also run clade model C (Bielawski and Yang 2004). We found signatures of divergent selection acting on the foreground branch (mammalian lineage, supplementary fig. 1, Supplementary Material online) on several genes (supplementary table S9, Supplementary Material online) but genes displaying signatures of positive selection (table 1) and divergent selection do not fully agree.

Expression Analysis of OHCs Positively Selected Genes We next determined by in situ hybridization (ISH) and immunohistochemistry (IC) the expression pattern only of those OHCs positively selected genes whose function in the inner ear is unknown. Thus, we did not include in this expression analysis the gene encoding prestin (SLC26A5) and Spectrin Beta I (SPTB). We also excluded from this analysis the genes ATPase Phospholipid Transporting 8 B1 (ATP8B1) and CLU. It has already been shown that ATP8B1 is specifically located in the stereocilia of the inner ear HCs and that a mutated version causes hearing loss associated with progressive degeneration of the cochlear HCs (Stapelbroek et al. 2009). In addition, a recent detailed analysis of CLU showed that it is expressed in supporting cells of the inner ear and not in the HCs (Lee et al. 2017). For the rest of the genes, we performed IC and/or ISH assays at P8, a stage where it have been previously shown many key genes display adultlike expression (Legendre et al. 2008; Son et al. 2012) and mechanotransduction is already present (Lelli et al. 2009). For selected genes, we performed also assays at P0. Our IHC assay shows that $L \bmod 3$ is strongly expressed in $\mathrm{OHCs}$ where it colocalizes with prestin, whereas no expression was found in $\mathrm{IHC}$ or in supporting cells (fig. $2 a-c$ ). Our ISH results indicate that Synm and General control of amino-acid synthesis 1 like 1 (Gcn1/1) genes are expressed in different cell types of the organ of Corti, including $\mathrm{OHCs}$, IHCs, and supporting cells; indicating 
Table 4. Outer Hair Cells Positively Selected Genes Extended Analysis.

\begin{tabular}{|c|c|c|c|c|c|c|c|}
\hline \multirow[b]{2}{*}{ ABLIM2 } & \multirow[t]{2}{*}{ Log Likelihood } & \multirow[t]{2}{*}{ LRT } & \multirow[t]{2}{*}{$P$} & \multicolumn{4}{|c|}{ Estimate of Parameters ${ }^{a}$} \\
\hline & & & & & & $50 \mathrm{spp}^{\mathrm{b}}$ & 663 sites $^{c}$ \\
\hline \multicolumn{8}{|l|}{ Mammals lineage } \\
\hline Positive hypothesis & $-28,230.44033$ & 4.03551 & 0.044552 & $p_{0}=0.91461$ & $p_{1}=0.07746$ & $p_{2 \mathrm{a}}=0.00731$ & $p_{2 \mathrm{~b}}=0.00062$ \\
\hline Null hypothesis & $-28,232.45808$ & & & $\omega_{0}=\mathbf{0 . 0 7 2 1 9}$ & $\omega_{1}=1.00000$ & $\omega_{2}=12.11450$ & $\omega_{2}=12.11450$ \\
\hline \multicolumn{8}{|l|}{ Birds lineage } \\
\hline Positive hypothesis & $-28,233.67308$ & 0.094326 & 0.75878 & $p_{0}=0.89578$ & $p_{1}=0.07656$ & $p_{2 a}=0.02548$ & $p_{2 b}=0.00218$ \\
\hline Null hypothesis & $-28,233.72024$ & & & $\omega_{0}=\mathbf{0 . 0 7 2 2 0}$ & $\omega_{1}=1.00000$ & $\omega_{2}=1.48360$ & $\omega_{2}=1.48360$ \\
\hline ATP8B1 & & & & & & & \\
\hline \multicolumn{8}{|l|}{ Mammals lineage } \\
\hline Positive hypothesis & $-76,919.307235$ & 8.08686 & 0.004459 & $p_{0}=0.85773$ & $p_{1}=0.11758$ & $p_{2 \mathrm{a}}=0.02171$ & $p_{2 b}=0.00298$ \\
\hline Null hypothesis & $-76,923.350665$ & & & $\omega_{0}=0.05846$ & $\omega_{1}=1.00000$ & $\omega_{2}=7.06785$ & $\omega_{2}=7.06785$ \\
\hline \multicolumn{8}{|l|}{ Birds lineage } \\
\hline Positive hypothesis & $-76,930.693283$ & 2.482594 & 0.115119 & $p_{0}=0.87567$ & $p_{1}=0.12055$ & $p_{2 a}=0.00333$ & $p_{2 \mathrm{~b}}=0.00046$ \\
\hline Null hypothesis & $-76,931.93458$ & & & $\omega_{0}=\mathbf{0 . 0 5 8 8 9}$ & $\omega_{1}=1.00000$ & $\omega_{2}=12.04765$ & $\omega_{2}=12.04765$ \\
\hline \multicolumn{8}{|l|}{ ATP13A1 } \\
\hline \multicolumn{8}{|l|}{ Mammals lineage } \\
\hline Positive hypothesis & $-67,283.355935$ & 23.760556 & $<0.00001$ & $p_{0}=0.89893$ & $p_{1}=0.07572$ & $p_{2 \mathrm{a}}=0.02338$ & $p_{2 \mathrm{~b}}=0.00197$ \\
\hline Null hypothesis & $-67,295.236213$ & & & $\omega_{0}=\mathbf{0 . 0 3 5 9 2}$ & $\omega_{1}=1.00000$ & $\omega_{2}=10.05311$ & $\omega_{2}=10.05311$ \\
\hline \multicolumn{8}{|l|}{ Birds lineage } \\
\hline Positive hypothesis & $-67,303.351861$ & 3.241314 & 0.071804 & $p_{0}=0.91056$ & $p_{1}=0.07767$ & $p_{2 a}=0.01084$ & $p_{2 \mathrm{~b}}=0.00092$ \\
\hline Null hypothesis & $-67,304.972518$ & & & $\omega_{0}=0.03603$ & $\omega_{1}=1.00000$ & $\omega_{2}=6.22608$ & $\omega_{2}=6.22608$ \\
\hline CLU & & & & & & $78 s p p^{b}$ & 501 sites $^{c}$ \\
\hline Mammals lineage & & & & & & & \\
\hline Positive hypothesis & $-39,005.226584$ & 4.236266 & 0.039571 & $p_{0}=0.80336$ & $p_{1}=0.16632$ & $p_{2 \mathrm{a}}=0.02513$ & $p_{2 \mathrm{~b}}=0.00520$ \\
\hline Null hypothesis & $-39,007.344717$ & & & $\omega_{0}=0.12843$ & $\omega_{1}=1.00000$ & $\omega_{2}=8.33236$ & $\omega_{2}=8.33236$ \\
\hline Birds lineage & & & & & & & \\
\hline Positive hypothesis & $-39,003.053931$ & 8.437492 & 0.003676 & $p_{0}=0.79636$ & $p_{1}=0.16745$ & $p_{2}=0.02991$ & $p_{2}=0.00629$ \\
\hline Null hypothesis & $-39,007.272677$ & & & $\omega_{0}=0.12812$ & $\omega_{1}=1.00000$ & $\omega_{2}=10.16714$ & $\omega_{2}=10.16714$ \\
\hline GCN1L1 & & & & & & 78 spp $^{b}$ & 2,703 sites $^{c}$ \\
\hline Mammals lineage & & & & & & & \\
\hline Positive hypothesis & $-14,4002.68408$ & 7.771752 & 0.005307 & $p_{0}=0.94045$ & $p_{1}=0.05581$ & $p_{2 \mathrm{a}}=0.00353$ & $p_{2 \mathrm{~b}}=0.00021$ \\
\hline Null hypothesis & $-14,4006.56995$ & & & $\omega_{0}=0.04724$ & $\omega_{1}=1.00000$ & $\omega_{2}=15.66888$ & $\omega_{2}=15.66888$ \\
\hline Birds lineage & & & & & & & \\
\hline Positive hypothesis & $-14,3998.659022$ & 1.10152 & 0.293937 & $p_{0}=0.92401$ & $p_{1}=0.05487$ & $p_{2 \mathrm{a}}=0.01994$ & $p_{2 b}=0.00118$ \\
\hline Null hypothesis & $-14,3999.209782$ & & & $\omega_{0}=0.04704$ & $\omega_{1}=1.00000$ & $\omega_{2}=1.87202$ & $\omega_{2}=1.87202$ \\
\hline LMOD3 & & & & & & 81 spp $^{b}$ & 766 sites $^{c}$ \\
\hline Mammals lineage & & & & & & & \\
\hline Positive hypothesis & $-47,870.657361$ & 6.891114 & 0.008663 & $p_{0}=0.68271$ & $p_{1}=0.28524$ & $p_{2 \mathrm{a}}=0.02260$ & $p_{2 b}=0.00944$ \\
\hline Null hypothesis & $-47,874.102918$ & & & $\omega_{0}=\mathbf{0 . 0 7 0 2 8}$ & $\omega_{1}=1.00000$ & $\omega_{2}=10.66286$ & $\omega_{2}=10.66286$ \\
\hline Birds lineage & & & & & & & \\
\hline Positive hypothesis & $-47,876.603126$ & 2.206312 & 0.137448 & $p_{0}=0.69522$ & $p_{1}=0.29308$ & $p_{2 a}=0.00823$ & $p_{2 \mathrm{~b}}=0.00347$ \\
\hline Null hypothesis & $-47,877.706282$ & & & $\omega_{0}=\mathbf{0 . 0 7 0 2 5}$ & $\omega_{1}=1.00000$ & $\omega_{2}=3.95284$ & $\omega_{2}=3.95284$ \\
\hline SLC26A5 & & & & & & $84 \mathrm{spp}^{\mathrm{b}}$ & 807 sites $^{c}$ \\
\hline Mammals lineage & & & & & & & \\
\hline Positive hypothesis & $-52,093.467526$ & 30.2245 & $<0.00001$ & $p_{0}=0.73431$ & $p_{1}=0.10694$ & $p_{2 a}=0.13857$ & $p_{2 \mathrm{~b}}=0.02018$ \\
\hline Null hypothesis & $-52,108.579776$ & & & $\omega_{0}=0.09071$ & $\omega_{1}=1.00000$ & $\omega_{2}=5.30036$ & $\omega_{2}=5.30036$ \\
\hline Birds lineage & & & & & & & \\
\hline Positive hypothesis & $-52,138.971652$ & 2.006386 & 0.156647 & $p_{0}=0.84820$ & $p_{1}=0.12910$ & $p_{2 a}=0.01970$ & $p_{2 \mathrm{~b}}=0.00300$ \\
\hline Null hypothesis & $-52,139.974845$ & & & $\omega_{0}=0.09195$ & $\omega_{1}=1.00000$ & $\omega_{2}=4.97296$ & $\omega_{2}=4.97296$ \\
\hline SPTB & & & & & & 44 spp $^{b}$ & 2,398 sites $^{c}$ \\
\hline Mammals lineage & & & & & & & \\
\hline Positive hypothesis & $-11,0358.198564$ & 27.037036 & $<0.00001$ & $p_{0}=0.87833$ & $p_{1}=0.08524$ & $p_{2 \mathrm{a}}=0.03321$ & $p_{2 \mathrm{~b}}=0.00322$ \\
\hline Null hypothesis & $-11,0371.717082$ & & & $\omega_{0}=0.06515$ & $\omega_{1}=1.00000$ & $\omega_{2}=5.91155$ & $\omega_{2}=5.91155$ \\
\hline Birds lineage & & & & & & & \\
\hline Positive hypothesis & $-11,0299.263432$ & 3.721744 & 0.05371 & $p_{0}=0.83473$ & $p_{1}=0.08077$ & $p_{2 \mathrm{a}}=0.07704$ & $p_{2 b}=0.00746$ \\
\hline Null hypothesis & $-11,0301.124304$ & & & $\omega_{0}=0.06360$ & $\omega_{1}=1.00000$ & $\omega_{2}=1.38252$ & $\omega_{2}=1.38252$ \\
\hline STRIP2 & & & & & & $81 \mathrm{spp}^{\mathrm{b}}$ & 901 sites $^{c}$ \\
\hline Mammals lineage & & & & & & & \\
\hline Positive hypothesis & $-46,610.73634$ & 20.10029 & $<0.00001$ & $p_{0}=0.87009$ & $p_{1}=0.09569$ & $p_{2 a}=0.03083$ & $p_{2 \mathrm{~b}}=0.00339$ \\
\hline Null hypothesis & $-46,620.78648$ & & & $\omega_{0}=\mathbf{0 . 0 3 9 8 1}$ & $\omega_{1}=1.00000$ & $\omega_{2}=10.64273$ & $\omega_{2}=10.64273$ \\
\hline Birds lineage & & & & & & & \\
\hline Positive hypothesis & $-46,643.009668$ & 4E-06 & 0.9984 & $p_{0}=0.89968$ & $p_{1}=0.10032$ & $p_{2 a}=0.00000$ & $p_{2 \mathrm{~b}}=0.00000$ \\
\hline Null hypothesis & $-46,643.00967$ & & & $\omega_{0}=\mathbf{0 . 0 4 0 6 1}$ & $\omega_{1}=1.00000$ & $\omega_{2}=1.00000$ & $\omega_{2}=1.00000$ \\
\hline
\end{tabular}


Table 4. Continued

\begin{tabular}{|c|c|c|c|c|c|c|c|}
\hline \multirow[b]{2}{*}{ SYNM } & \multirow[t]{2}{*}{ Log Likelihood } & \multirow[t]{2}{*}{ LRT } & \multirow[t]{2}{*}{$P$} & \multicolumn{4}{|c|}{ Estimate of Parameters ${ }^{a}$} \\
\hline & & & & & & $76 \mathrm{spp}^{\mathrm{b}}$ & 1,878 sites $^{c}$ \\
\hline \multicolumn{8}{|l|}{ Mammals lineage } \\
\hline Positive hypothesis & $-13,4254.028601$ & 132.065834 & $<0.00001$ & $p_{0}=0.54280$ & $p_{1}=0.28765$ & $p_{2 a}=0.11082$ & $p_{2 b}=0.05873$ \\
\hline Null hypothesis & $-13,4320.061518$ & & & $\omega_{0}=0.22981$ & $\omega_{1}=1.00000$ & $\omega_{2}=999.00000$ & $\omega_{2}=999.00000$ \\
\hline \multicolumn{8}{|l|}{ Birds lineage } \\
\hline Positive hypothesis & $-13,4282.805668$ & 33.644914 & $<0.00001$ & $p_{0}=0.53697$ & $p_{1}=0.28892$ & $p_{2 \mathrm{a}}=0.11320$ & $p_{2 b}=0.06091$ \\
\hline Null hypothesis & $-13,4299.628125$ & & & $\omega_{0}=0.22770$ & $\omega_{1}=1.00000$ & $\omega_{2}=6.29011$ & $\omega_{2}=6.29011$ \\
\hline ZNF516 & & & & & & 74 spp $^{b}$ & 1,388 sites $^{c}$ \\
\hline \multicolumn{8}{|l|}{ Mammals lineage } \\
\hline Positive hypothesis & $-95,494.724183$ & 19.631404 & $<0.00001$ & $p_{0}=0.84625$ & $p_{1}=0.12654$ & $p_{2 a}=0.02366$ & $p_{2 b}=0.00354$ \\
\hline Null hypothesis & $-95,504.539885$ & & & $\omega_{0}=0.12131$ & $\omega_{1}=1.00000$ & $\omega_{2}=13.47129$ & $\omega_{2}=13.47129$ \\
\hline \multicolumn{8}{|l|}{ Birds lineage } \\
\hline Positive hypothesis & $-95,508.679517$ & 2.395726 & 0.121669 & $p_{0}=0.86921$ & $p_{1}=0.13020$ & $p_{2 \mathrm{a}}=0.00051$ & $p_{2 b}=0.00008$ \\
\hline Null hypothesis & $-95,509.87738$ & & & $\omega_{0}=0.12199$ & $\omega_{1}=1.00000$ & $\omega_{2}=116.17970$ & $\omega_{2}=116.17970$ \\
\hline
\end{tabular}

${ }^{\mathrm{a}} \omega$ values shown for each site class correspond to foreground branch.

${ }^{\mathrm{b}}$ The number of vertebrate species analyzed.

${ }^{\mathrm{c}}$ Number of codons analyzed.

that they are not $\mathrm{HC}$ specific (fig. $2 d-i)$. In contrast, ISH assays for ATPase 13A1 (Atp13a1) (fig. 2j-l) and for Zinc finger protein 516 (Znf516) (fig. 2m-o) failed to detect these genes to be expressed in the organ of Corti at the developmental stage analyzed (P8). In addition, our results indicated that Strip2 is strongly expressed in OHCs, in IHCs and also in the spiral ganglion at P8 (fig. $2 p-s$ ). We also observed that Strip2 expression is strong in the spiral ganglion but more diffuse in the organ of Corti at P0 (supplementary fig. S5, Supplementary Material online). We found that Ablim2 is expressed in both, OHCs and IHCs at P8 (fig. 2t-v) but not at PO (supplementary fig. $55 d$ and e, Supplementary Material online). In the spiral ganglion, it is strongly expressed at both stages analyzed (fig. $2 t$ and $w$ and supplementary fig. S5d and $f$, Supplementary Material online). It is important to note that our expression analysis on positively selected genes in $\mathrm{OHCs}$ revealed some discrepancies with the original database where these genes were obtained. Liu et al. (2014) examined the transcriptome of manually collected IHCs and OHCs from adult mouse cochleae and using microarray they reported 1,193 IHCs and $198 \mathrm{OHCs}$ genes that were differentially expressed $(P<0.05)$ in one cell population over the other. Therefore, this database does not inform if these genes are expressed in other cellular types of the inner ear. This explains why we found Ablim2, Strip2, Gon1/1, and Synm to be also expressed in the spiral ganglion and in supporting cells, respectively.

Altogether, these results allowed us to select those genes expressed in IHCs and/or OHCs, for follow-up in-depth evolutionary and functional studies. Taking into account our evolutionary and expression analysis results together, we decided to further study STRIP2 and ABLIM2 since their participation in inner ear function is unknown.

\section{Comprehensive Evolutionary Analysis and Functional Characterization of STRIP2}

To better understand the impact of the evolutionary remodeling on STRIP2, we identified particular amino acids displaying the signature of positive selection in mammals. To do that, after applying the branch-site positive selection test to the basal branch of mammals (fig. 3a), we performed a follow-up Bayesian analysis that calculates the posterior probabilities that a site is under adaptive evolution. Our results show a strong signal of adaptive molecular evolution in the mammalian lineage in 11 sites (fig. $3 b$ and c). Four of these positively selected amino acids were located in the N1221 domain, an acidic polypeptide with several possible transmembrane regions which function is still unknown (fig. 3c).

To generate Strip 2 mutant mice, we used a single guide RNA directed to exon 1 that induced a 64-bp deletion, which predicts the generation of an inactive truncated protein (fig. 3c). We verified the generation of Strip2 mutations in the engineered mice by genomic DNA sequencing. Absence of Strip2 protein expression was confirmed by Western blot analysis (fig. 3d). We observed a lack of Strip2 protein in the hearts of Strip2 $2^{-1-}$ mice (fig. 3d), a tissue where Strip2 is strongly expressed (Eden et al. 2016). The mRNA encoding Strip2 is detected in mutant mice (supplementary fig. S6, Supplementary Material online) since the probe used in ISH assays is directed to exon 4, which is not affected by the mutation generated.

We performed auditory functional studies of Strip2 newly generated mutant mice by means of two complementary techniques that allow differential diagnosis of OHCs versus $\mathrm{IHC} /$ neuronal dysfunction throughout the cochlea. To evaluate the integrity of the hearing system, we recorded auditory brainstem responses (ABRs) that are sound-evoked potentials generated by neuronal circuits in the ascending auditory pathways (Kujawa and Liberman 2009). We also evaluated the OHCs function through distortion product otoacoustic emissions (DPOAEs) testing (Shera and Guinan 1999).

We measured DPOAEs thresholds at different frequencies (from 5.6 to $45.25 \mathrm{kHz}$ ) in Strip $2^{-/-},{ }^{+/-}$, and ${ }^{+/+}$littermates at 2 months. Remarkably, there were no changes in DPOAEs thresholds in the three genotypes (Strip $2^{+/+} n=8$; Strip $2^{+/-}$ $n=18$; and Strip $2^{-1-} n=7$; fig. 3g). These results show that the lack of Strip2 does not affect the OHCs' biological motors. 

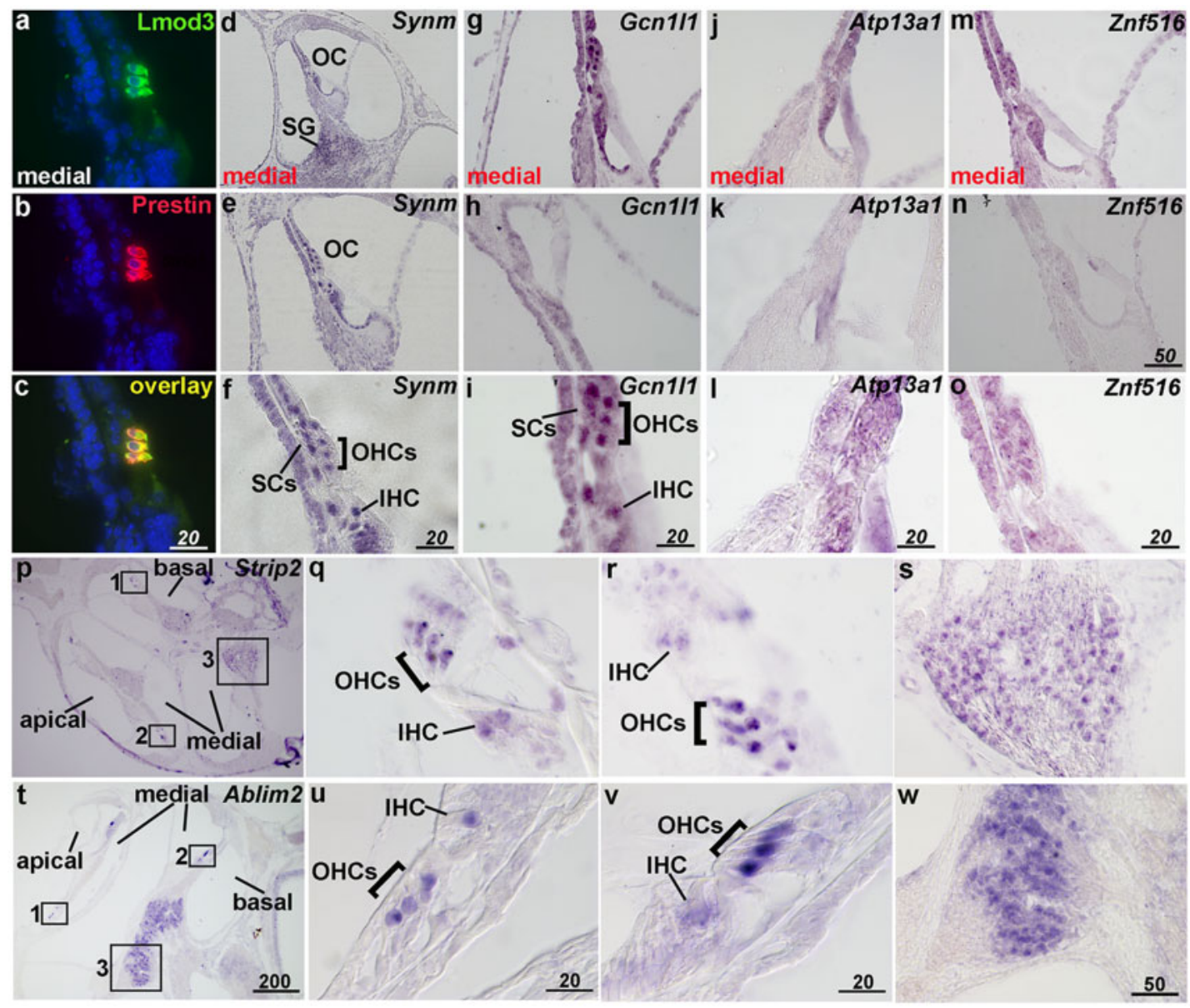

FIG. 2. Expression analysis of OHCs positively selected genes in the inner ear. (a) Photomicrographs of IC assays showing Lmod3 expression, (b) prestin expression, and (c) colocalization of these two proteins in OHCs. (d) ISH showing that Synm is expressed in the spiral ganglion and in different cellular types in the organ of Corti $(e, f)$, including OHCs and IHCs. $(g) G c n 1 / 1$ expression in the organ of Corti is localized in several cellular types. ( $h$ ) Results obtained using the Gcn1/1 sense probe. (i) A magnification of Gcn1/1 expression showing IHCs, OHCs, and SCs in the organ of Corti. (j) Atp13a1 expression in the organ of Corti obtained with the antisense probe $(k)$ and with the sense probe. (I) A magnification of Atp13a1 expression showing IHCs, OHCs, and SCs in the organ of Corti. $(m)$ Znf516 expression in the organ of Corti obtained with the antisense probe and $(n)$ with the sense probe. (o) A magnification of Znf516 expression showing IHCs, OHCs, and SCs in the organ of Corti. ( $p$ ) Low-magnification photomicrograph showing Strip2 differential expression in OHCs and IHCs in the organ of Corti across different turns of the cochlea and in the spiral ganglion $(q, r)$. Higher magnification of the section across the organ of Corti indicated by the squares 1 and 2. (s) Higher magnification of the spiral ganglion indicated by square 3. $(t)$ Low-magnification photomicrograph showing Ablim2 differential expression in OHCs and IHCs in the organ of Corti across different turns of the cochlea and in the spiral ganglion. $(u, v)$ Higher magnification of the section across the organ of Corti indicated by the squares 1 and 2. $(w)$ Higher magnification of the spiral ganglion indicated by square 3 .

In addition, we found no differences in ABR thresholds at all the frequencies tested (Strip2 ${ }^{+/+} n=8$; Strip2 ${ }^{+/-} n=18$; and Strip $2^{-1-} n=7$; fig. 3h). However, when analyzing ABR wave $\mathrm{l}$, we found a large reduction in amplitudes at 8,16 , and $32 \mathrm{kHz}$ in both Strip2 $2^{-1-}$ and ${ }^{+/-}$mice $\left(\right.$Strip2 ${ }^{+/+} n=8$; Strip $^{+l-} n=18$; and Strip $2^{-1-} n=7$; fig. 3i). These results might indicate a potential cochlear neuropathy. The $A B R$ wave I latencies were not altered in the different genotypes, suggesting that the lack of Strip2 does not affect cochlear nerve conduction. Through ISH analysis we verified that Strip2 is expressed at the cochlear nucleus in the brain (CN; supplementary fig. S7, Supplementary Material online). However, since no alteration in wave II ABRs amplitudes were observed, no overt malfunction of synaptic transmission at this central relay is expected.
To analyze if the reduction in ABR peak I amplitude is due to the loss of auditory-nerve synapses, we immunostained whole-mount organs of Corti with antibodies against CtBP2-Ribeye, a critical protein present at the presynaptic ribbon (Khimich et al. 2005), and GluA2 AMPA-type glutamate receptors, which are expressed at the postsynaptic afferent terminal (Matsubara et al. 1996). In each immunostained whole-mount organ of Corti, we counted the colocalized synaptic puncta at the medial, apical, and basal cochlear regions. We found that the number of synaptic puncta per IHC was significantly reduced in the three regions analyzed (basal Strip2 ${ }^{+/+} 12.73 \pm 0.2944$ and Strip2 $2^{-1-}$ $8.729 \pm 0.5265 \quad t=6.251 \quad \mathrm{df}=233 \quad P<0.001 ;$ medial Strip2 $^{+/+} 13.74 \pm 0.2209$ Strip2 $^{-/-} \quad t=10.37 \mathrm{df}=278$ $P<0.001 ;$ apical Strip $2^{+/+} 11.27 \pm 0.3958$, Strip $2^{-1-}$ 
a

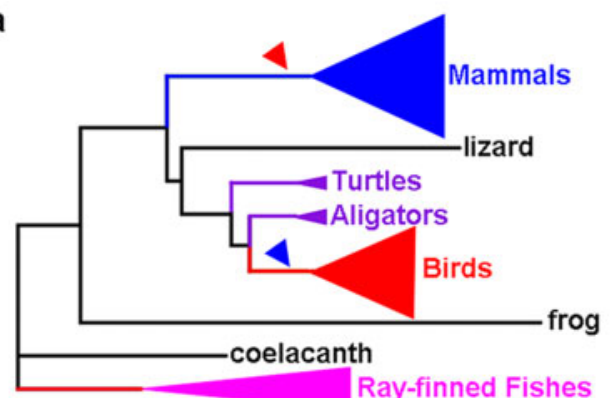

C
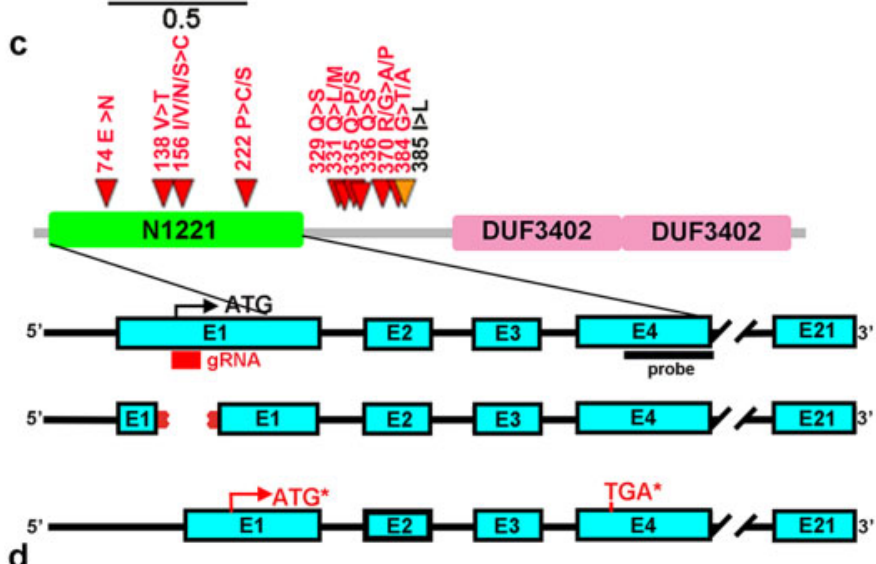

d
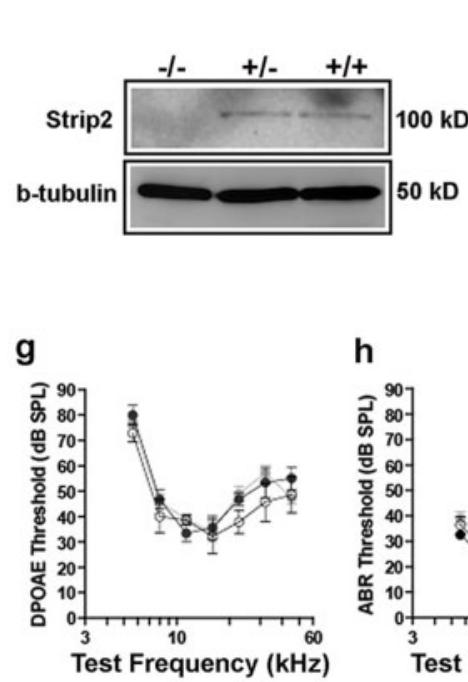
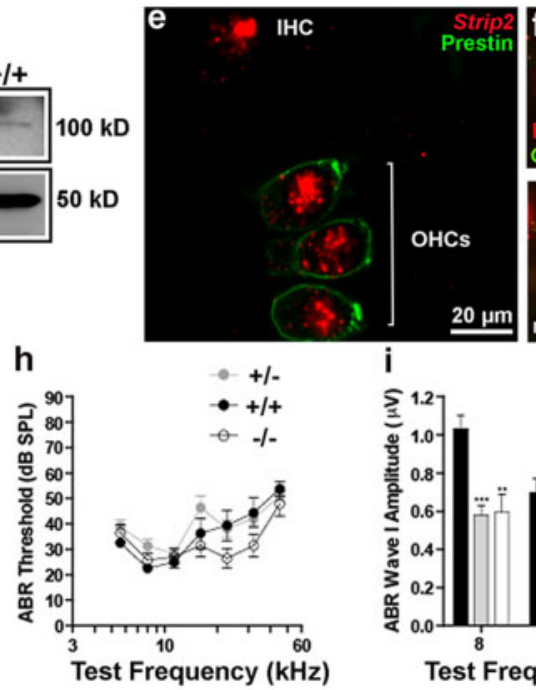

b
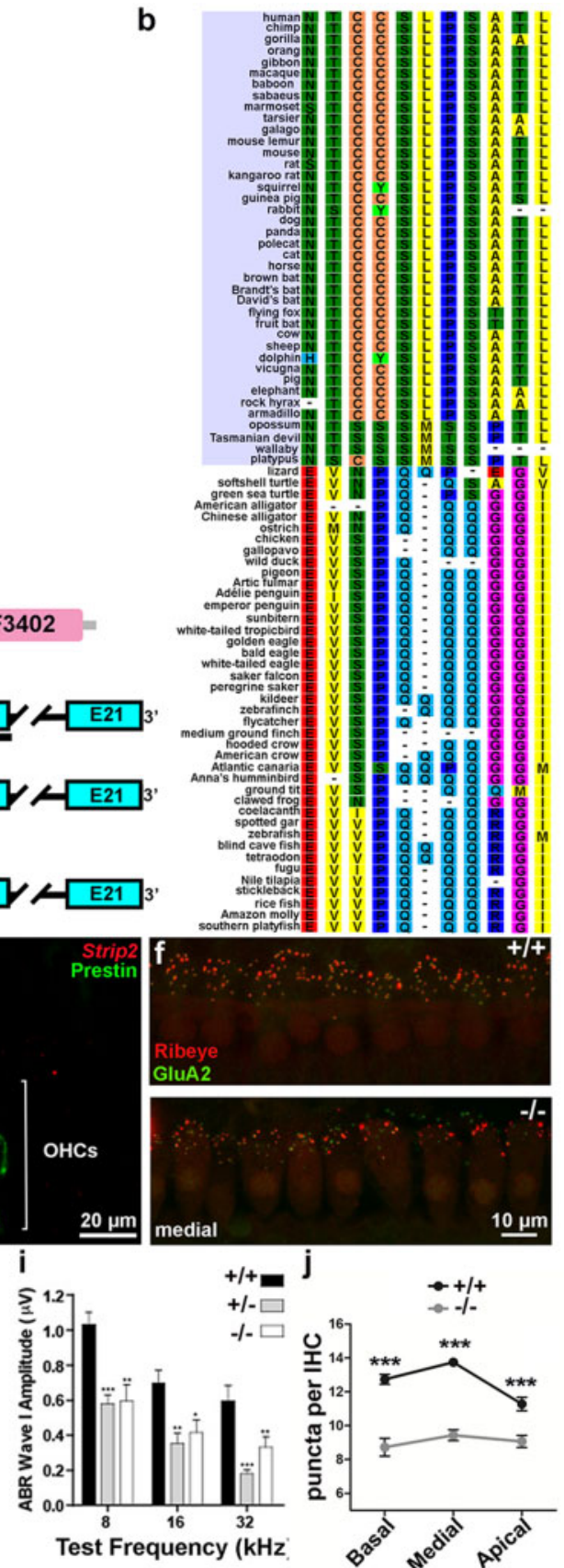

FIG. 3. STRIP2 is expressed in OHCs and IHCs, evolved adaptively in the mammalian lineage and it is important for IHCs function. (a) Phylogenetic tree of the 81 analyzed vertebrate species where some lineages are shown as collapsed branches for clarity. The mammalian and bird lineages (focal branches of the evolutionary analysis) are indicates with red and blue arrows, respectively. (b) Detail of the positively selected sites in mammals showing the changes in amino acids in the different vertebrates analyzed. (c) Top: Schematic drawing of the Strip2 protein showing the different functional domains. Red and yellow arrows indicate the location in the different functional domains of the positively selected sites in the mammalian lineage. Bottom: Scheme of the strategy developed to generate the mutant mice pedigree lacking Strip2. The sites of priming of the guide RNA on exon 1 and for the probe used for ISH on exon 4 are indicated. The deleted region affecting this exon is also shown. This deletion predicts the creation of an additional initiation codon (ATG*) a premature STOP codon (TGA*) in exon 4. (d) Western blot showing strong Strip2 expression in wild type mouse heart samples $\left(\right.$ Strip $2^{+/+}$) a tissue where Strip2 is strongly expressed (Eden et al. 2016). We observed lack of expression in Strip $2^{-1-}$. (e) Photomicrographs of ISH and IC assays showing the expression of Strip2 and prestin in IHCs and OHCs in mouse cochleas. $(f)$ Representative confocal images of IHCs synapses from the medial turn of the cochleae immunolabeled for presynaptic ribbons (CtBP2-red) and postsynaptic receptor patches (GluA2-green) in Strip $2^{-/-}$and ${ }^{+/+}$. Anti-CtBP2 antibody also weakly stains IHC nuclei. ( $g$ ) DPOAEs and $(h)$ ABRs thresholds measurements in Strip $2^{-/-},{ }^{+/-}$, and ${ }^{+/+}$in 2-month-old mice at different frequencies (from 5.6 to $45.25 \mathrm{kHz}$ ). (i) ABR wave I amplitude at 8,16 , and $32 \mathrm{kHz}$. Statistical analysis: one-way ANOVA followed by Tukey's multiple comparison test; ${ }^{*} P<0.05$; ${ }^{* *} \mathrm{P}<0.01$; and ${ }^{* * *} \mathrm{P}<0.001$. (j) Synapsis quantification and statistical analysis. 
$9.066 \pm 0.3580 t=4.095 \mathrm{df}=157 \mathrm{P}<0.001 ; n=5$ for both genotypes; mean \pm SEM; fig. $3 f$ and $j$ ).

\section{Comprehensive Evolutionary Analysis and Functional Characterization of ABLIM2}

In order to identify amino acids sites displaying signatures of positive selection, we performed an exhaustive evolutionary analysis using $50 \mathrm{ABLIM} 2$ vertebrate sequences (fig. $4 a$ and $c$ ). Our analysis, identified two amino acids ( $518 \mathrm{~L} / \mathrm{P}>\mathrm{S} ; 557 \mathrm{E} /$ $A>N$ ) that displayed signatures of positive selection, both located in the Adherens-junction domain (fig. $4 b$ and $c$ ).

Since it has been reported that Ablim2 interacts with $\mathrm{F}$ actin (Barrientos et al. 2007), we analyzed the evolution of genes encoding actin in the inner ear (ACTB and ACTG; Drummond et al. 2012) in the mammalian lineage. We found no signatures of positive selection in any of these genes in the lineage leading to mammals $(P=1$; supplementary table $S 10$, Supplementary Material online).

In addition, to assess if $\mathrm{F}$-actin and Ablim2 colocalized in $\mathrm{HCs}$ of the mammalian cochlea, we performed immunostaining of both proteins. Our results indicated that Ablim2 is mainly distributed in the cytoplasm and that both proteins colocalized at the stereocilia of inner and outer HCs (fig. 4f).

Mutant mice lacking Ablim2 were generated by deleting 202 base pairs comprising the last 48 amino acids of exon 2 and $58 \mathrm{bp}$ of the second intron (fig. 4b). The induced mutation was confirmed by genomic sequencing and it predicts the appearance of a premature stop codon and the generation of an inactive truncated protein. Immunohistochemical analysis confirmed that Ablim2 protein is absent in the inner ear of Ablim2 $2^{-1-}$ mice (fig. $4 d$ and $e$ ), whereas the mRNA encoding Ablim2 is detected in mutant mice (supplementary fig. S8, Supplementary Material online) since the probe used in ISH assays includes exon 3-20, which are not affected by the mutation generated.

We analyzed the auditory function in mice lacking Ablim2 and found that cochlear thresholds were not affected when measured by DPOAEs or ABRs (Ablim2 ${ }^{+/+} n=11$; Ablim2 $^{+/-} n=18$; and Ablim2 $2^{-1-} n=8$; fig. $4 \mathrm{~g}$ and $h$ ). The earliest wave of the ABR (wave I), which represents the summed sound-evoked spike activity at the first synapse between IHCs and afferent nerve fibers, was not modified in amplitude or latency in Ablim2 $2^{-1-}$ mice at 8,16 , or $32 \mathrm{kHz}$ (Ablim2 $^{+/+} n=11$; Ablim2 $^{+/-} n=18$; and Ablim2 ${ }^{-1-} n=8$; fig. 4i). These results suggest that the absence of Ablim2 does not affect either cochlear amplification or auditory-nerve function.

\section{Discussion}

To unravel the evolutionary history of the mammalian auditory system, we performed a detailed evolutionary analysis of several databases reporting genes expressed in the inner ear. We analyzed more than 1,300 coding gene sequences and identified 165 displaying signatures of positive selection in the mammalian lineage. Since we are particularly interested in the evolution of the cochlea in mammals, we focused our analysis in genes expressed in OHCs and IHCs. We surprisingly found that a similar proportion of $\mathrm{IHCs}$ and $\mathrm{OHCs}$ genes underwent positive selection, suggesting that both cellular types underwent significant functional remodeling in the mammalian lineage. We selected two of these genes, ABLIM2 and STRIP2, to perform functional studies that included the generation of mutant mouse strains lacking these proteins. We discovered that Strip $2^{-1-}$ and Strip2 $2^{+-}$mice exhibited a large reduction in ABR peak I amplitudes, suggesting that Strip2 plays a functional role in the first synapse between IHCs and afferent nerve fibers. Moreover, cochlear sensory epithelium of Strip $2^{-1-}$ immunostained for pre- and postsynaptic markers showed a reduction in auditory-nerve synapses, suggesting that lack of Strip2 expression leads to cochlear synaptopathy.

The evolutionary analysis of OHCs genes identified 11 genes showing strong signatures of positive selection in the mammalian lineage. This might aid to unravel the evolution of the genetic network that shaped the remarkable functional motor capacities of this mammalian cell type. These include genes such as SLC26A5 (encoding prestin) and SPTB (erythrocytic spectrin beta 1 ) previously identified as key players in the evolution of the mammalian inner ear, which underwent selective pressure in mammals and are expressed in $\mathrm{OHCs}$ (Franchini and Elgoyhen 2006; Okoruwa et al. 2008; Elgoyhen and Franchini 2011; Cortese et al. 2017).

Within the 198 OHCs differentially expressed genes, some particular genes previously shown to be strongly expressed in $\mathrm{OHCs}$ and also to have undergone positive selection in the mammalian lineage were absent. These include the nicotinic acetylcholine receptor subunit alpha 10 (Chrna10), and Spectrin Beta, Non-Erythrocytic 5 (Sptbn5) (Franchini and Elgoyhen 2006; Elgoyhen and Franchini 2011; Cortese et al. 2017). Although Chrna10 was reported as the most differentially expressed gene in OHCs (Liu et al. 2014), this gene was filtered out in our automatic pipeline because it has been duplicated in the fish lineage (Franchini and Elgoyhen 2006). However, we have previously carried out deep evolutionary studies with this nicotinic receptor and shown that it underwent positive selection and functional remodeling in the mammalian lineage (Franchini and Elgoyhen 2006; Elgoyhen and Franchini 2011; Lipovsek et al. 2012). On the other hand, since Sptbn5 is expressed at similar levels in both, $\mathrm{IHCs}$ and $\mathrm{OHCs}$, it was not found as differentially expressed in one cell type over the other (Liu et al. 2014). To our surprise, Sptbn 5 was not reported in the other two HC expression databases analyzed in this study (Burns et al. 2015; Scheffer et al. 2015).

Our data highlight that several OHCs genes underwent positive selection probably underlying some of the morphological and functional changes that this cellular type experienced throughout mammalian evolution. In addition, it is interesting to note that we found a similar proportion of genes expressed in IHCs that went through adaptive evolution in the mammalian lineage. However, our expression analysis shows that most of the genes previously reported to be differentially expressed in OHCs (Liu et al. 2014), are in fact strongly expressed in IHCs and other cell types in the mammalian inner ear. Our results suggest that careful expression 
a
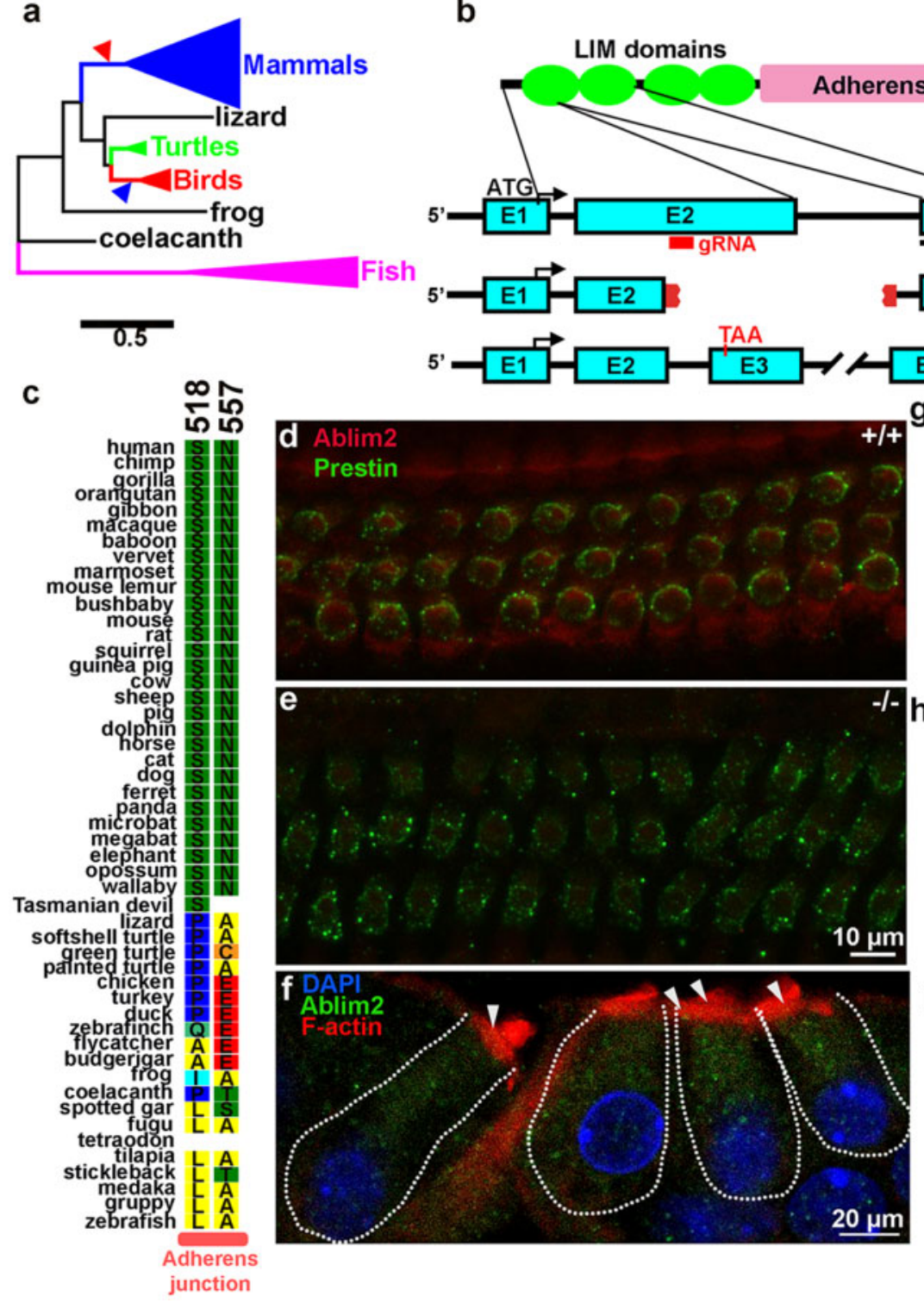

b

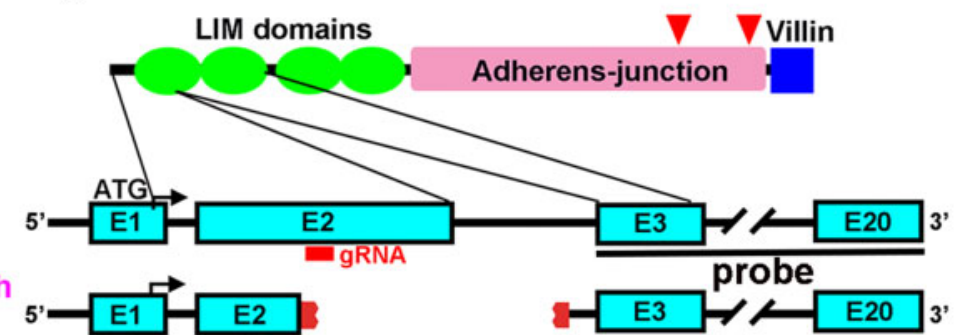

E20 3'

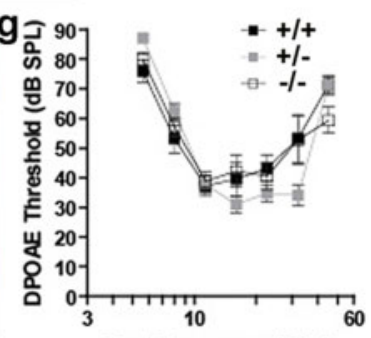

Test Frequency $(\mathbf{k H z})$
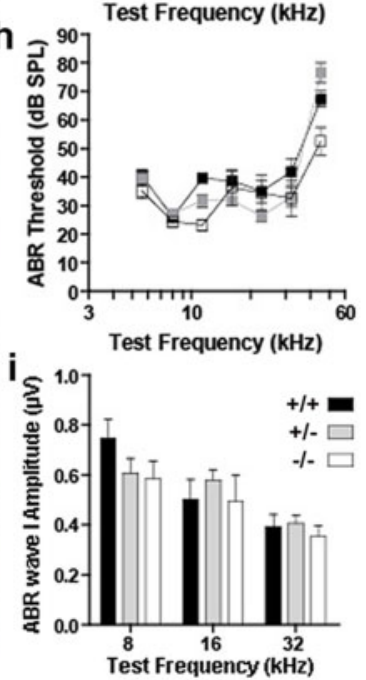

FIG. 4. ABLIM2 is expressed in OHCs and IHCs and evolved adaptively in the mammalian lineage. (a) Phylogenetic tree of the 50 analyzed vertebrate species where some lineages are shown as collapsed branches for clarity. The mammalian and bird lineages (focal branches of the evolutionary analysis) are indicated with red and blue arrows, respectively. (b) Top: Schematic drawing of the ABLIM2 protein showing the different functional domains. Red arrows indicate the location in the Adherens-junction domain of the positively selected sites in the mammalian lineage. Bottom: Scheme of the strategy developed to generate the mutant mice pedigree lacking Ablim2. The site of priming of the guide RNA on exon 2 and for the probe used for ISH is indicated. The deleted region affecting exon 2 is also shown. This deletion is predicted to generate a premature STOP codon in exon 3. (c) Detail of the amino acids positively selected in the different vertebrates analyzed. $(d, e)$ IC assays showing the expression of Ablim 2 and prestin in Ablim2 $2^{-/-}$and ${ }^{+/+}$representative mice. $(f) I C$ showing expression of Ablim2, F-actin, and DAPI in OHCs and IHCs. White arrows indicate colocalization spots in the stereocilia. (g) DPOAEs (top) and (h) ABRs (bottom) thresholds measurements in Ablim2 $2^{-/-},{ }^{+/-}$, and ${ }^{+/+}$in $2-$ month- $^{-}$ old mice at different frequencies (from 5.6 to $45.25 \mathrm{kHz}$ ). (i) ABR wave I amplitude at 8,16 , and $32 \mathrm{kHz}$. Statistical analysis: one-way ANOVA followed by Tukey's multiple comparison test.

analyses should be performed in order to be able to identify genes uniquely expressed in IHCs and OHCs. Our findings also indicate that many genes expressed in both, $\mathrm{IHCs}$ and $\mathrm{OHCs}$ underwent evolutionary driven genetic remodeling. This massive molecular make over might underlie the profound division of labor that IHCs and OHCs play in the mammalian cochlea in comparison to mainly undifferentiated vestibularlike HCs probably present in the basilar papilla of reptilian mammalian ancestors. OHCs function in the cochlea is to magnify the stimulus, amplifying both the amplitude and frequency selectivity of basilar membrane vibration for lowlevel sounds (Dallos 2008; Dallos et al. 2008; Ashmore et al. 2010). This function is dependent on somatic electromotility mediated by prestin (Zheng et al. 2000; Dallos and Fakler 2002). In contrast, IHCs behave as true sensory cells and probably maintained a more similar role to HCs present in the ancestral basilar papilla (Elliott et al. 2018). Division of labor has also been observed in HCs of the avian auditory 
papilla (the equivalent to the cochlea in mammals), which possess tall (THCs) and short hair cells (SHCs) (Murrow and Fuchs 1990). However, current data indicate that even though SHCs and THCs in the chicken auditory papilla show analogies with OHCs and IHCs, they differentiate in several aspects: prestin is highly expressed in both cellular types, and voltageinduced bundle motion is not confined to the SHCs since it is also observed in THCs (Beurg et al. 2013). Thus, the mammalian specific division of labor between IHCs and OHCs is consistent with the extensive molecular adaptations that we have uncovered and that most probably underlie the functional specialization of these mammalian phenotypical novelties. Thus, our data provide a new framework for analyzing the evolution of the mammalian inner ear in which adaptively driven changes in genes expressed in both, $\mathrm{IHCs}$ and $\mathrm{OHCs}$ occurred in order to accommodate the specific and exclusive functions of each cellular type. However, the scarce availability of gene expression studies in more primitive mammalian forms such as monotremes and marsupials and in other nonmammalian vertebrates limit our understanding of how this division of labor and specialization appeared along mammalian evolution since it is hypothesized that the reptile lineage that originated mammals possessed a basilar papilla where HCs were maybe more undifferentiated.

Taking into account our evolutionary findings and the gene expression study outcome, we selected ABLIM2 and STRIP2 to perform a comprehensive assessment of positive selection in vertebrates and functional analysis through the generation of null mutant mice where we examined auditory function. Our results indicated that both, ABLIM2 and STRIP2 displayed signatures of adaptive evolution in mammals but not in the lineage leading to birds (Aves), suggesting that other proteins could underlie the evolutionary changes observed in the inner ear in this group of vertebrates. In addition, both ABLIM2 and STRIP2 evolved adaptively in the tetrapod lineage suggesting that they might also be important for the remodeling of the inner ear that led to the appearance of the basilar papilla, the precursor to the organ of Corti (for a review see Fritzsch et al. [2013]).

Our functional studies indicated that Strip2 (also known as FAM $40 B$ or Myoscape) is important in inner ear physiology since mice lacking Strip2 show an impaired first synapse between IHCs and afferent nerve fibers as evidenced by a reduction of the number of afferent synaptic contacts and of the ABR peak I amplitudes. It has been recently shown that Strip2 directly interacts with the C-terminal tail domain of the L-type $\mathrm{Ca}^{2+}$ channel in cardiomyocytes (Eden et al. 2016). Knockdown of Strip2 in cardiomyocytes results in downregulation of $\mathrm{Ca}^{2+}$ channel expression together with a reduction of calcium channel currents (Eden et al. 2016). In the inner ear, HC neurotransmission is thought to mainly rely on $\mathrm{Ca}_{v}$ 1.3 L-type $\mathrm{Ca}^{2+}$ channels (Fuchs and Evans 1990; Roberts et al. 1990; Moser and Beutner 2000; Platzer et al. 2000; Spassova et al. 2001). In addition, it has been proposed that $\mathrm{L}$-type $\mathrm{Ca}^{2+}$ channels may have a role in $\mathrm{HC}$ development. Before the onset of hearing in altricial rodents, IHCs fire $\mathrm{Ca}^{2+}$ action potentials (Kros et al. 1998; Glowatzki and Fuchs 2000; Beutner and Moser 2001), which drive afferent synaptic transmission (Kros et al. 1998; Glowatzki and Fuchs 2000; Beutner and Moser 2001; Glowatzki and Fuchs 2002). This presensory activity is probably important for the development and maintenance of the auditory pathway (Tierney et al. 1997; Mostafapour et al. 2000). Mice lacking Cav1.3 channels are deaf and finally undergo degeneration of afferent auditory-nerve fibers and HCs (Brandt et al. 2003). We can hypothesize that, in the inner ear, Strip2 may interact with $\mathrm{Cav}_{\mathrm{v}} 1.3$ channels promoting the correct assembling and/or the maintenance of auditory-nerve synapses. It would be interesting to investigate if at the onset of hearing the proper number of synapses per IHCs is formed and degenerate afterward or if they never developed. Nonetheless, our results show that despite the loss of auditory-nerve synapses, ABRs thresholds were not elevated, a clear indication of cochlear neuropathy (Furman et al. 2013), indicating that Strip2 might be important for the survival of low-spontaneous rate auditory-nerve fibers.

It is interesting to note that Strip2 plays a role in cell shape and cytoskeleton organization (Bai et al. 2011) and that the functional properties of OHCs are largely based in a highly specialized cytoskeleton linked to the cytoplasmic membrane that allows their remarkable conformational changes (Ashmore et al. 2010). Moreover, our functional annotation analysis indicates that genes linked to cytoskeleton functions are overrepresented among OHCs positively selected genes. The fact that DPOAEs thresholds were normal in Strip2 $2^{-1-}$ mice indicates that the lack of this protein does not affect the OHCs' electromotile function.

On the other hand, our functional studies indicated that mice lacking Ablim2 display normal cochlear thresholds, as measured by both, DPOAEs and ABRs. These results might suggest that Ablim2 does not participate in cochlear amplification or in IHCs function or that other functionally redundant gene could compensate its absence. Our expression pattern analysis of Ablim2 in the inner ear indicates that it is highly expressed in OHCs and IHCs of the organ of Corti, and also in the spiral ganglion. ABLIM2 is a STARS (striated muscle activator of Rho signaling) protein that strongly binds to F-actin (Barrientos et al. 2007). It has been shown that Ablim2 is highly expressed in muscle where, it apparently regulates the sensing of biomechanical stress and also in the brain where it participates in axon guidance (Barrientos et al. 2007). Our colocalization studies indicate that Ablim2 is expressed in the cytoplasm of HCs and it seems to colocalize with F-actin at the stereocilia. Since ACTB and ACTG, actinencoding genes, did not evidence signatures of positive selection, they did not undergo parallel evolution with ABLIM2. Further functional studies will be necessary to determine the functional relevance of this protein in inner ear physiology.

It is interesting to analyze our results in the light of one of the most fundamental debates in adaptive evolution: does evolution proceed primarily through changes in proteincoding DNA or in noncoding regulatory sequences? It has been hypothesized that morphological adaptation occurs mainly via noncoding changes, since many developmental genes are active in many tissues, whereas noncoding mutations can modify a gene's activity in just one or two tissues, 
avoiding pleiotropic effects (Carroll 2008). In contrast, it has been also suggested that adaptive evolution of proteincoding sequences, as well as other mechanisms like gene duplication, play a significant role in the evolution of form and function (Hoekstra and Coyne 2007). In addition, a combination of these two mechanisms seems to also play a role through the duplication of genes followed by subfunctionalization (i.e., duplicated genes or paralogs, retain a subset of its original ancestral function or acquire different expression domains) (Lynch and Force 2000; Kaessmann 2010). In this regard, the co-option of new regulatory machinery seems to be the mechanism that facilitates the acquisition of novel expression domains (for a review see Peter and Davidson [2011]). In the evolution of the inner ear, the several steps that led to the appearance of the complex sensory organ of vertebrates (Fritzsch and Elliott 2017) must have probably involved all these evolutionary mechanism acting on many genes and gene regulatory networks. Therefore, more comparative analysis about the evolution of protein-coding and noncoding sequences that control the expression of genes in the developing and adult inner ear would be necessary to better illuminate our understanding of functional and morphological evolution of this mammalian sensory organ.

In summary, through this evolutionary approach we discovered that STRIP2 underwent strong positive selection in the mammalian lineage and plays an important role in the physiology of the inner ear. Moreover, our combined evolutionary and functional studies allow us to speculate that the extensive evolutionary remodeling that this gene underwent in the mammalian lineage provided an adaptive value. Thus, our study is a proof of concept that evolutionary approaches paired with functional studies could be a useful tool to uncover new key players in the function of organs and tissues.

\section{Materials and Methods}

\section{Sequences Retrieval}

Selected species for this study were chosen in order to maximize the presence of reported orthologs for the majority of genes analyzed, taking care to keep a balance between mammalian and nonmammalian vertebrate species. Our default chosen species for this study were: human (Homo sapiens), mouse (Mus musculus), dog (Canis familiaris), opossum (Monodelphis domestica), chicken (Gallus gallus), anole lizard (Anolis carolinensis), and zebrafish (Danio rerio). This reduced number of well-annotated species allowed us to assemble small multisequence alignments suitable to carry out short exploratory analysis of our inner ear databases. The list of TFs expressed in the inner ear was assembled through bibliographic searches (supplementary table \$4, Supplementary Material online). Orthologs finding and coding sequence download were performed through BioMart data mining tool, consulting Ensembl orthologs database (releases 80 through 89). As BioMart downloads one sequence per coding sequence reported for each gene in each species, only the longest coding sequence was kept as criteria to choose that which would best represent the coding sequence of the complete locus. In order to perform an evolutionary analysis on a gene, this must be represented by only one, ortholog in each species. Therefore, two filtering steps were carried on to take away, first those genes for which an ortholog could not be found in one or more of the selected species, and second, those genes that presented orthology relationships of the type one-to-many. Thus a subset of the original genes, suitable to be automatically processed and analyzed, was defined. For deeper analysis of individual genes, coding sequences of all available vertebrate species were downloaded from Ensembl (www.ensembl.org; last accessed April 21, 2019), low quality sequences were excluded and visualization and manual curation of the multiple species alignments were performed in MEGA5 (Tamura et al. 2011), using mainly GenBank Nucleotide (www.ncbi.nlm.nih.gov/genbank; last accessed April 21, 2019) sequence reports. Species tree for each deeply analyzed gene were pruned using ETE Toolkit Python framework (Huerta-Cepas et al. 2010, 2016) from the Ensembl full species tree.

\section{Sequence Alignment}

For the high-throughput analysis, sequence alignments were performed with transAlign (Bininda-Emonds 2005) in order to keep codon-reading frame in DNA sequence. Additionally, a Perl script called batchAlign was implemented to carry out the sequence alignments of multiple genes, in batch (available through Bininda-Emonds at http://www.uni-oldenburg.de/ en/biology/systematics-and-evolutionary-biology/; last accessed April 21, 2019). For deeper analysis of individual genes, sequence alignments were performed using Clustal W implemented in MEGA5 software (Tamura et al. 2011) and manually curated to detect and fix alignment errors.

\section{Evolutionary Analysis}

To carry out the adaptive evolutionary analysis, the modified Model A branch-site test 2 of positive selection (Zhang et al. 2005) was applied in codeml program from PAML4 (version 4.2) package (Yang 2007). Two-nested hypotheses were tested for the focal lineage: the alternative hypothesis in which positive selection was allowed only in the selected branch and the null hypothesis where no positive selection was allowed. In the alternative hypothesis three $\omega$ values $(\omega$ is the relation among nonsynonymous and synonymous changes fixation rates $[\omega=\mathrm{d} N / \mathrm{d} S])$ are calculated: $\omega_{0}, \omega_{1}$, and $\omega_{2}$ and four proportions of site classes: $p_{0}, p_{1}, p_{2 \mathrm{a}}$ and $p_{2 \mathrm{~b}}$ are calculated for the codons under negative, null, and positive selection, respectively. Although $\omega_{1}$ is fixed to 1 in this model, $\omega_{0}$ and $\omega_{2}$ must be estimated for the alternative hypothesis. In the null hypothesis, as no positively selected sites are allowed and $\omega_{2}$ is also fixed to 1 , only $\omega_{0}$ estimate is used. The posterior probabilities of the given data to fit the proposed evolutionary model in each hypothesis were obtained by maximum likelihood optimization. Likelihood ratio tests (LRTs) were performed from such probability values comparing twice the difference of the log-likelihood probabilities for both hypotheses against a chi-square distribution with 1 degree of freedom. In those cases where the positive selection test rejected the null hypothesis, a posteriori Bayesian approach to specifically identify the sites under positive 
selection along the branch of the tree studied was implemented by codeml after maximum likelihood estimations were concluded. This Bayes Empirical Bayes analysis estimates the posterior probability of each site in the alignment to belong to each of the site classes determined by the model (Zhang et al. 2005).

In the seven species batch exploratory analysis, as a complete deletion parameter was set, an additional filter was added before the analysis that excluded those genes with such a high proportion of gaps and missing information in their sequence alignments that they could not be processed by PAML. Hereafter, we implemented a method to simplify and lower the computational time required to perform the branch-site positive selection analysis in PAML: we split the optimization of the total number of parameters in this complex model of positive selection test running first the $M 0$ basic model over the species raw topology and obtained the optimized maximum likelihood branch lengths using the Goldman and Yang (1994) codon-based evolutionary model of DNA evolution. Once the branch lengths were estimated, the obtained phylogeny was assigned as the fixed input tree that was used without modifications throughout the branchsite positive selection test. In this way, the branch lengths of the species tree, which comprises an important number of the estimated parameters, are precalculated in the $M 0$ run, leaving only the model specific parameters to be optimized during the branch-site test. This approach has shown to be very accurate (Yang $Z$, personal communication), and its results highly consistent among reruns of the same data. Our multiPAML in-house Python program not only mimics this approach but also automatizes it to make scalable to large quantity of genes. This program also complements the analysis applying a multiple testing correction to the resulting $P$ values through the Benjamini and Hochberg method, commonly known as false discovery rate (Benjamini and Hochberg 1995). The number of positively selected genes is informed based on corrected $P$ values, a greater number of genes displaying signatures of positive selection were found when considering uncorrected $P$ values (supplementary tables S11 and S12, Supplementary Material online).

For deeper analysis of individual genes, in particular those preferentially expressed in $\mathrm{OHCs}$ and previously identified to be under positive selection in the mammalian basal lineage, all available vertebrate species were used (supplementary table S13, Supplementary Material online) and other three focal branches were tested: birds basal lineage (Aves), tetrapods basal lineage (Tetrapoda), and tetrapods plus Coelacanth (Latimeria chalumnae) lineages.

We used PROVEAN (Choi et al. 2012) to predict the potential functional impact of positively selected sites in the mammalian lineage for the deeply analyzed OHCs differentially expressed genes. Amino acid substitutions were considered as "deleterious" if the PROVEAN score was $\leq-2.5$. We considered amino acid substitutions as "neutral" if the PROVEAN score was $>-2.5$.

In order to test the presence of different selection pressures acting on different clades, we used Clade Model C (Bielawski and Yang 2004). Clade Model C allows three site classes, two of them are always under purifying or neutral selection and the third site class is allowed to vary between foreground and background branches. Following Davies et al. (2012), we performed a LRT comparing it to the M1a model (Nearly Neutral), a sites model that does not allow variation among clades. For the LRT, we used 3 degrees of freedom (df), for the difference in the number of estimated parameters between these two models.

\section{In Situ Hybridization}

For ISH in cryosections, P0, P8, and adult (2-month-old) mice were anesthetized and perfused using $4 \%$ paraformaldehyde (PFA) in phosphate-buffered saline (PBS) at $4^{\circ} \mathrm{C}$. Cochleas from at least three mice were dissected and fixed in 4\% PFA in PBS at $4{ }^{\circ} \mathrm{C}$ overnight (ON). Cochleas were then treated with a solution containing $0.5-\mathrm{mM}$ ethylenediaminetetraacetic acid (EDTA) pH 8 in PBS for 3 days at $4^{\circ} \mathrm{C}$, incubated in $30 \%$ sucrose-PBS solution overnight at $4^{\circ} \mathrm{C}$ and embedded in optimal cutting temperature (ThermoFisher Scientific). Blocks were frozen for $1 \mathrm{~min}$ in isopentane at $-70^{\circ} \mathrm{C}$ and stored at $-80^{\circ} \mathrm{C}$. Serial $10-\mu \mathrm{m}$-thick sections were cut in a cryostat (Leica 1510S, Germany), collected on Super-Frost Plus slides (FisherScientific, USA), and stored at $-80^{\circ} \mathrm{C}$ until use. Gene coding sequences were cloned into plasmids (pGEMteasy, PROMEGA) through PCR on CDNA using specific primers (supplementary table S9, Supplementary Material online). To synthetize RNA probes, plasmids containing (Strip2; Znf516; Ablim2; Atp13a1; Gcn1/1; Synm; and $L \bmod 3)$ were linearized using restriction enzymes, and transcribed using T7 RNA polymerase or SP6 (Roche, Penzberg, Germany) in order to generate digoxigenin (DIG)-labeled sense and antisense riboprobes. After thawing sections, they were postfixed with 4\% PFA in PBS for $10 \mathrm{~min}$ and then rinsed with PBS for $15 \mathrm{~min}$. Sections were acetylatilated in agitation for $10 \mathrm{~min}(\mathrm{HCl} 36 \%$, acetic anhydride $0.25 \% \mathrm{v} / \mathrm{v}$, triethanolamine $1.36 \% \mathrm{v} / \mathrm{v}$ ), followed by permeabilization for $30 \mathrm{~min}$ (1\% Triton X-100 in PBS). After washing in PBS, prehybridization was carried out at room temperature for $3-4 \mathrm{~h}$ in a solution containing $50 \%$ formamide, $10 \%$ dextran sulfate, $5 \times$ Denhardt's solution, and $250-\mathrm{mg} / \mathrm{ml}$ tRNA. Hybridization was performed with $300 \mathrm{ng} / \mathrm{ml}$ of the probe in hybridization solution at $72{ }^{\circ} \mathrm{C}$ for $16 \mathrm{~h}$. Thereafter, samples were washed with $0.2 \%$ saline-sodium citrate at $72{ }^{\circ} \mathrm{C}$ for $45 \mathrm{~min}$ to $1 \mathrm{~h}$ and then twice for $5 \mathrm{~min}$ with B1 solution (containing $100-\mathrm{mM} \mathrm{NaCl}, 0.1 \%$ Triton $\mathrm{X}-100$, and $100-\mathrm{mM}$ Tris- $\mathrm{HCl} \mathrm{pH} \mathrm{7.5).} \mathrm{After} \mathrm{blocking} \mathrm{with} 10 \%$ normal goat serum in $\mathrm{B} 1$ solution for $4 \mathrm{~h}$, the sections were incubated $\mathrm{ON}$ with alkaline phosphatase-conjugated anti DIG Fab fragments (Roche; 1:3,500 in B1). The next day, sections were washed in $\mathrm{B} 1$ and incubated in $\mathrm{B} 2(100-\mathrm{mM} \mathrm{NaCl}, 50-\mathrm{mM} \mathrm{MgCl}$, $0.1 \%$ Tween, and $100-\mathrm{mM}$ Tris- $\mathrm{HCl} \mathrm{pH} \mathrm{9.5)} \mathrm{twice} \mathrm{for} 10 \mathrm{~min}$. The time of the staining reaction began when $\mathrm{B} 2+$ NBT-BCIP (Roche, $0.2 \% \mathrm{v} / \mathrm{v}$ ) reactive was added. Samples were checked after $1 \mathrm{~h}$, and every $20 \mathrm{~min}$ under a microscope to determine when the detection reaction should be stopped. The reaction was stopped when the signal to noise ratio was at an optimum. Both sense and antisense reactions were stopped at the same time. Finally, samples were coverslipped with a solution 
containing polyvinyl alcohol and glycerol in Tris $0.2 \mathrm{M}, \mathrm{pH} 8.5$. ISH samples were photographed using a microscope Leica DM2500 coupled to a digital camera Leica DFC $7000 \mathrm{~T}$. Images were processed using Adobe Photoshop.

For fluorescent ISH, after blocking with $10 \%$ normal goat serum in B1 solution for $4 \mathrm{~h}$, the sections were incubated $\mathrm{ON}$ with horseradish peroxidase-conjugated anti DIG Fab fragments (Roche; 1:500 in B1). The next day, sections were washed in B1 and incubated in Cy3 Amplification Reagent Working Solution (Perkin Elmer) for 30 min. Finally, samples were coverslipped with a solution containing Vectashield mounting medium (Vector Laboratories, USA). Samples were photographed using a Confocal Leica TCS SPE microscope and images were processed using Adobe Photoshop.

\section{Mutant Mice Generation}

Mouse strains carrying deleted (knockout) alleles were created using a modified CRISPR/Cas9 protocol (Wang et al. 2013). Briefly, sgRNA recognition sequence targeting the Ablim2 region ( $5^{\prime}$-agaacaagtacttccacatc AGG-3', where AGG is the PAM) or the Strip2 region ( $5^{\prime}$-atggacgaccccgc ggcacc GGG-3', where GGG is the PAM) were designed using CRISPR Design Tool (http://crispr.mit.edu/; last accessed April $21,2019)$. No potential off-targets were found by searching for matches in the mouse genome $(\mathrm{mm} 10)$ and allowing for up to two mismatches in the 20-nt sequence preceding the NGG PAM sequence. The T7 promoter was added to the recognition sequence, and the whole sgRNA was generated by a PCR with a reverse primer $\left(5^{\prime}\right.$-aaaagcaccgactcggtgcc- $\left.3^{\prime}\right)$ from the pX330 plasmid. The T7-sgRNA product was used as a template for in vitro transcription using the MEGAshortscript T7 kit (ThermoFisher Scientific). The Cas9 mRNA was in vitro transcribed from pMLM3613 plasmid using the mMESSAGE mMACHINE T7 kit (Thermo Fisher Scientific) and polyadenylated using Poly(A) Tailing Kit (Thermo Fisher Scientific \#AM1350). Knockout mice were generated by microinjecting $1 \mathrm{pl}$ of a solution containing Cas9 mRNA (final concentration of $100 \mathrm{ng} / \mu \mathrm{l})$ and sgRNA $(50 \mathrm{ng} / \mu \mathrm{l})$ in nuclease-free water directly into the cytoplasm of FVB/NJ zygotes and a few hours later transferred to the oviduct of pseudopregnant FVB/NJ females. F0 mice were genotyped by PCR (see genotyping in supplementary table S14, Supplementary Material online) and mutations identified by DNA sequencing. All analyses were performed in littermates resulting of F1 heterozygous mattings (F2). All mouse procedures were performed in agreement with the INGEBI-CONICET Laboratory Animal Welfare and Research Committee.

\section{Cochlear Function Tests}

To evaluate the integrity of the hearing system, we recorded ABRs that are sound-evoked potentials generated by neuronal circuits in the ascending auditory pathways (Kujawa and Liberman 2009). We also evaluated the OHCs function through DPOAEs testing, which can be measured from the external auditory canal. When two tones are presented to the normal ear, distortion components at additional frequencies are produced in the $\mathrm{HC}$ receptor potentials that can drive the OHCs' electromotility to move the sensory epithelium at the distortion frequencies. The resulting pressure waves from the motion of the sensory epithelium are conducted back to the eardrum to produce DPOAEs, which can be measured in the ear canal (Shera and Guinan 1999). ABRs and DPOAEs were performed on mice anesthetized with xylazine $(20 \mathrm{mg} / \mathrm{kg}$, i.p.) and ketamine $(100 \mathrm{mg} / \mathrm{kg}$, i.p.) and placed in an acoustically electrically shielded chamber maintained at $30^{\circ} \mathrm{C}$. Recordings were performed at postnatal day 60 (P60). Sound stimuli were delivered through a custom acoustic system with two dynamic earphones used as sound sources (CDMG1500803A; CUI) and an electret condenser microphone (FG23329-PO7; Knowles) coupled to a probe tube to measure sound pressure near the eardrum (for details, see https:// www.masseyeandear.org/research/otolaryngology/investigators/laboratories/eaton-peabody-laboratories/epl-engineering-resources/epl-acoustic-system; last accessed April 21, 2019). Digital stimulus generation and response processing were handled by digital I-O boards from National Instruments driven by custom software written in LabVIEW. For ABRs, needle electrodes were placed into the skin at the dorsal midline close to the neural crest and pinna with a ground electrode near the tail. ABR potentials were evoked with 5 -ms tone pips (0.5-ms rise-fall, with a $\cos ^{2}$ envelope, at 40/s) delivered to the eardrum at log-spaced frequencies from 5.6 to $45.25 \mathrm{kHz}$. The response was amplified $10,000 \times$ with a $0.3-3$ $\mathrm{kHz}$ passband. Sound level was raised in $5-\mathrm{dB}$ steps from 10 to 80-dB sound pressure level. At each level, 1,024 responses were averaged with stimulus polarity alternated. The DPOAEs in response to two primary tones of frequency $f_{1}$ and $f_{2}$ were recorded at $\left(2 \times f_{1}\right)-f_{2}$, with $f_{2} / f_{1}=1.2$, and the $f_{2}$ level $10 \mathrm{~dB}$ lower than the $f_{1}$ level. Ear-canal sound pressure was amplified and digitally sampled at $4-\mu$ s intervals. DPOAE threshold was defined as the lowest $f_{2}$ level in which the signal to noise floor ratio is $>1$.

\section{Cochlear Processing and Immunostaining}

Cochleae from 2-month-old mice (P60) were extracted, perfused intralabyrinthly with 4\% PFA in PBS, postfixed with $4 \%$ PFA ON and decalcified in 0.12-M EDTA for 5 days. Cochlear tissues were then microdissected and permeabilized by freeze/thawing in 30\% sucrose (for CtBP2/GluA2 immunostaining) or directly blocked (for Ablim/prestin immunostaining). The microdissected pieces were blocked in 5\% normal goat serum (for CtBP2/GluA2 immunostaining) or $5 \%$ normal donkey serum (for Ablim/prestin immunostaining) with 1\% Triton $\mathrm{X}-100$ in PBS for $1 \mathrm{~h}$, followed by incubation in primary antibodies (diluted in blocking buffer) at $37^{\circ} \mathrm{C}$ for $16 \mathrm{~h}$ (for CtBP2/GluA2 immunostaining) or $4^{\circ} \mathrm{C}$ for $16 \mathrm{~h}$ (for Ablim/ prestin immunostaining). The primary antibodies used in this study were 1) rabbit anti-ablim antibody (rabbit polyclonal anti-Ablim2, Abcam ab100926; 1:200), 2) goat anti-prestin antibody (Santa Cruz Biotechnology Inc. sc22692; 1:700), 3) anti-C-terminal binding protein 2 (mouse anti-CtBP2 IgG1; BD Biosciences, San Jose, CA; 1:200), and 4) anti-glutamate receptor 2 (mouse anti-GluA2 IgG2a; Millipore, Billerica, MA; 1:2,000). For F-actin detection we used TRITC-Phalloidin (Sigma). Tissues were then incubated with the appropriate Alexa Fluor-conjugated fluorescent secondary antibodies 
(Invitrogen, Carlsbad, CA; 1:1,000 in blocking buffer) for $2 \mathrm{~h}$ at room temperature. Finally, tissues were mounted on microscope slides in FluorSave mounting media (Millipore, Billerica, $M A)$. Confocal z-stacks (0.2- $\mu \mathrm{m}$ step size) of the medial region from each cochlea were taken using a Leica TCS SPE microscope equipped with $63 \times(1.5 \times$ digital zoom $)$ oil-immersion lens. To check for organ of Corti aberrant morphology in Strip2 $^{-1-}$ mice whole-mount preparations were immunostained with prestin antibody and observed under the microscope in comparison to wild type littermates. No signals of aberrant morphology was observed (supplementary fig. S9, Supplementary Material online).

\section{Western Blot Analyses}

To analyze Strip2 expression in mutated and wild type mice, we performed western blot assays using heart and liver protein extracts prepared in a protein extraction buffer $(50-\mathrm{mM}$ Tris- $\mathrm{HCl}$ pH 7.5, 2-mM EDTA; 1\% Triton X-100; 150-mM $\mathrm{NaCl} ; 0.05 \%$ SDS; Halt Protease and Phosphatase Inhibitor Cocktail $[100 \times]$ [ Thermo Scientific 78440]). Then, we added $150-\mathrm{mM} \mathrm{NaCl}, 0.2 \%$ glycerol, $2 \%$ bromophenol blue, and $\beta$ mercaptoethanol to $10 \%$ to the protein extract and heated them at $100^{\circ} \mathrm{C}$ ( $5 \mathrm{~min}$ ). Samples were then separated on $12 \%$ SDS-polyacrylamide gels (prepared with acrilamide and $\mathrm{N} \mathrm{N}^{\prime}$ methylenebisacrilamide $30 \%$, Invitrogen) and transferred using a wet transfer to nitrocellulose membranes (BIO-RAD). Membranes were blocked in $5 \%(\mathrm{w} / \mathrm{v})$ nonfat dry milk, $0.05 \%$ $v / v$ Tween 20 in TBS (milk/1xTBS-T) for $1 \mathrm{~h}$. After blocking, membranes were incubated $\mathrm{ON}$ at $4^{\circ} \mathrm{C}$ in blocking solution containing the primary antibody. The membrane was probed with a polyclonal anti-human STRIP2 antibody produced in rabbit (HPA019657 Sigma) at a dilution of 1:500 according to the manufacturer's protocol. After washing three times in TBS containing $0.05 \% \mathrm{v} / \mathrm{v}$ Tween 20 , blots were incubated with the appropriate secondary antibody donkey anti-rabbit HRP conjugate (1:2,000, Fisher Scientific) for $3 \mathrm{~h}$ at room temperature. Polyclonal anti-human beta Tubulin antibody produced in rabbit (Abcam Ab6046) was used as loading control at a dilution 1:500. Proteins were visualized using ECL detection (Cell Signaling Technology SignalFire ECL Reagent \#6883) and by exposing on the GeneGnomeXRQ (Syngene).

\section{Supplementary Material}

Supplementary data are available at Molecular Biology and Evolution online.

\section{Author Contributions}

L.F.F. designed and supervised the project. F.P., J.M.S., A.R.C., V.C.C., M.E.G.-C., and L.F.F. conducted experiments and data analyses. L.F.F. wrote the manuscript. M.R., A.B.E., and M.E.G.$C$. discussed experiments, provided reagents and materials, and edited the manuscript. All authors edited and approved the final version of this report.

\section{Acknowledgments}

We thank Marta Treimun for superb technical assistance with mutant mice generation. This work was supported by grants from the Agencia Nacional de Promoción Científica y
Tecnológica (PICT2013-1642, PICT2015-1726, and PICT2016-1429) to L.F.F., F.P., J.M.S., and A.R.C. have doctoral fellowship from the Consejo Nacional de Investigaciones Científicas y Técnicas (CONICET-Argentina). V.C.C. has a University of Buenos Aires fellowship for students.

\section{References}

Ashmore J, Avan P, Brownell WE, Dallos P, Dierkes K, Fettiplace R, Grosh K, Hackney CM, Hudspeth AJ, Julicher F, et al. 2010. The remarkable cochlear amplifier. Hear Res. 266(1-2):1-17.

Ashmore JF. 1987. A fast motile response in guinea-pig outer hair cells: the cellular basis of the cochlear amplifier. J Physiol. 388:323-347.

Bai SW, Herrera-Abreu MT, Rohn JL, Racine V, Tajadura V, Suryavanshi N, Bechtel S, Wiemann S, Baum B, Ridley AJ. 2011. Identification and characterization of a set of conserved and new regulators of cytoskeletal organization, cell morphology and migration. BMC Biol. 9:54.

Barrientos T, Frank D, Kuwahara K, Bezprozvannaya S, Pipes GC, BasselDuby R, Richardson JA, Katus HA, Olson EN, Frey N. 2007. Two novel members of the ABLIM protein family, ABLIM-2 and -3 , associate with STARS and directly bind F-actin. I Biol Chem. 282(11):8393-8403.

Belyantseva IA, Adler HJ, Curi R, Frolenkov GI, Kachar B. 2000. Expression and localization of prestin and the sugar transporter GLUT-5 during development of electromotility in cochlear outer hair cells. J Neurosci. 20(24):RC116.

Benjamini Y, Hochberg Y. 1995. Controlling the false discovery rate: a practical and powerful approach to multiple testing. J R Stat Soc Ser B Methodol. 57(1):289-300.

Bernstein P. 2003. The ear region of Latimeria chalumnae: functional and evolutionary implications. Zoology (Jena) 106(3):233-242.

Beurg M, Tan X, Fettiplace R. 2013. A prestin motor in chicken auditory hair cells: active force generation in a nonmammalian species. Neuron 79(1):69-81.

Beutner D, Moser T. 2001. The presynaptic function of mouse cochlear inner hair cells during development of hearing. J Neurosci. 21(13):4593-4599.

Bielawski JP, Yang Z. 2004. A maximum likelihood method for detecting functional divergence at individual codon sites, with application to gene family evolution. J Mol Evol. 59(1):121-132.

Bininda-Emonds OR. 2005. transAlign: using amino acids to facilitate the multiple alignment of protein-coding DNA sequences. BMC Bioinformatics 6:156.

Bouchard M, de Caprona D, Busslinger M, Xu P, Fritzsch B. 2010. Pax2 and Pax8 cooperate in mouse inner ear morphogenesis and innervation. BMC Dev Biol. 10:89.

Brandt A, Striessnig J, Moser T. 2003. CaV1.3 channels are essential for development and presynaptic activity of cochlear inner hair cells. J Neurosci. 23(34):10832-10840.

Brownell WE, Bader CR, Bertrand D, de Ribaupierre Y. 1985. Evoked mechanical responses of isolated cochlear outer hair cells. Science 227(4683):194-196.

Burns JC, Kelly MC, Hoa M, Morell RJ, Kelley MW. 2015. Single-cell RNASeq resolves cellular complexity in sensory organs from the neonatal inner ear. Nat Commun. 6:8557.

Carroll SB. 2008. Evo-devo and an expanding evolutionary synthesis: a genetic theory of morphological evolution. Cell 134(1):25-36.

Choi Y, Sims GE, Murphy S, Miller JR, Chan AP. 2012. Predicting the functional effect of amino acid substitutions and indels. PLoS One 7(10):e46688.

Cortese M, Papal S, Pisciottano F, Elgoyhen AB, Hardelin JP, Petit C, Franchini LF, El-Amraoui A. 2017. Spectrin betaV adaptive mutations and changes in subcellular location correlate with emergence of hair cell electromotility in mammalians. Proc Natl Acad Sci U S A. 114(8):2054-2059.

Dallos P. 2008. Cochlear amplification, outer hair cells and prestin. Curr Opin Neurobiol. 18(4):370-376. 
Dallos P, Fakler B. 2002. Prestin, a new type of motor protein. Nat Rev Mol Cell Biol. 3(2):104-111.

Dallos P, Wu X, Cheatham MA, Gao J, Zheng J, Anderson CT, Jia S, Wang $X$, Cheng WHY, Sengupta S, et al. 2008. Prestin-based outer hair cell motility is necessary for mammalian cochlear amplification. Neuron 58(3):333-339.

Davies KT, Cotton JA, Kirwan JD, Teeling EC, Rossiter SJ. 2012. Parallel signatures of sequence evolution among hearing genes in echolocating mammals: an emerging model of genetic convergence. Heredity (Edinb). 108(5):480-489.

Drummond MC, Belyantseva IA, Friderici KH, Friedman TB. 2012. Actin in hair cells and hearing loss. Hear Res. 288(1-2):89-99.

Duncan JS, Fritzsch B. 2013. Continued expression of GATA3 is necessary for cochlear neurosensory development. PLoS One 8(4):e62046.

Eden $M$, Meder B, Volkers M, Poomvanicha M, Domes K, Branchereau M, Marck P, Will R, Bernt A, Rangrez A, et al. 2016. Myoscape controls cardiac calcium cycling and contractility via regulation of L-type calcium channel surface expression. Nat Commun. 7:11317.

Elgoyhen AB, Franchini LF. 2011. Prestin and the cholinergic receptor of hair cells: positively-selected proteins in mammals. Hear Res. 273(12):100-108.

Elliott KL, Fritzsch B, Duncan JS. 2018. Evolutionary and developmental biology provide insights into the regeneration of organ of Corti hair cells. Front Cell Neurosci. 12:252.

Franchini LF, Elgoyhen AB. 2006. Adaptive evolution in mammalian proteins involved in cochlear outer hair cell electromotility. Mol Phylogenet Evol. 41(3):622-635.

Fritzsch B. 1987. Inner ear of the coelacanth fish Latimeria has tetrapod affinities. Nature 327(6118):153-154.

Fritzsch B, Elliott KL. 2017. Evolution and development of the inner ear efferent system: transforming a motor neuron population to connect to the most unusual motor protein via ancient nicotinic receptors. Front Cell Neurosci. 11:114.

Fritzsch B, Pan N, Jahan I, Duncan JS, Kopecky BJ, Elliott KL, Kersigo J, Yang T. 2013. Evolution and development of the tetrapod auditory system: an organ of Corti-centric perspective. Evol Dev. 15(1):63-79.

Fuchs PA, Evans MG. 1990. Potassium currents in hair cells isolated from the cochlea of the chick. J Physiol (Lond). 429(1):529-551.

Furman AC, Kujawa SG, Liberman MC. 2013. Noise-induced cochlear neuropathy is selective for fibers with low spontaneous rates. J Neurophysiol. 110(3):577-586.

Glowatzki E, Fuchs P. 2002. Transmitter release at the hair cell ribbon synapse. Nat Neurosci. 5(2):147-154.

Glowatzki E, Fuchs PA. 2000. Cholinergic synaptic inhibition of inner hair cells in the neonatal mammalian cochlea. Science 288(5475):2366-2368.

Goldman N, Yang Z. 1994. A codon-based model of nucleotide substitution for protein-coding DNA sequences. Mol Biol Evol. 11(5):725-736.

Grothe B, Carr EC, Casseday JH, Fritzsch B, Köppl C. 2004. The evolution of central pathways and their neural processing patterns. In: Manley GA, Fay RR, Popper AN, editors. Evolution of the vertebrate auditory system. New York: Springer. p. 289-359.

Hoekstra HE, Coyne JA. 2007. The locus of evolution: evo devo and the genetics of adaptation. Evolution 61(5):995-1016

Huang da W, Sherman BT, Lempicki RA. 2009. Systematic and integrative analysis of large gene lists using DAVID bioinformatics resources. Nat Protoc. 4(1):44-57.

Huerta-Cepas J, Dopazo J, Gabaldon T. 2010. ETE: a python Environment for Tree Exploration. BMC Bioinformatics 11:24.

Huerta-Cepas J, Serra F, Bork P. 2016. ETE 3: reconstruction, analysis, and visualization of phylogenomic data. Mol Biol Evol. 33(6):1635-1638.

Kaessmann H. 2010. Origins, evolution, and phenotypic impact of new genes. Genome Res. 20(10):1313-1326.

Khimich D, Nouvian R, Pujol R, Tom Dieck S, Egner A, Gundelfinger ED, Moser T. 2005. Hair cell synaptic ribbons are essential for synchronous auditory signalling. Nature 434(7035):889-894.
Kopecky BJ, Decook R, Fritzsch B. 2012. N-Myc and L-Myc are essential for hair cell formation but not maintenance. Brain Res. 1484:1-14.

Kros CJ, Ruppersberg JP, Rusch A. 1998. Expression of a potassium current in inner hair cells during development of hearing in mice. Nature 394(6690):281-284.

Kruger M, Schmid T, Kruger S, Bober E, Braun T. 2006. Functional redundancy of NSCL-1 and NeuroD during development of the petrosal and vestibulocochlear ganglia. Eur J Neurosci. 24(6):1581-1590.

Kujawa SG, Liberman MC. 2009. Adding insult to injury: cochlear nerve degeneration after "temporary" noise-induced hearing loss. J Neurosci. 29(45):14077-14085.

Ladhams A, Pickles JO. 1996. Morphology of the monotreme organ of Corti and macula lagena. J Comp Neurol. 366(2):335-347.

Lee S, Shin JO, Sagong B, Kim UK, Bok J. 2017. Spatiotemporal expression patterns of clusterin in the mouse inner ear. Cell Tissue Res. 370(1):89-97.

Legendre K, Safieddine S, Kussel-Andermann P, Petit C, El-Amraoui A. 2008. alphall-betaV spectrin bridges the plasma membrane and cortical lattice in the lateral wall of the auditory outer hair cells. J Cell Sci. 121(Pt 20):3347-3356.

Lelli A, Asai Y, Forge A, Holt JR, Geleoc GS. 2009. Tonotopic gradient in the developmental acquisition of sensory transduction in outer hair cells of the mouse cochlea. J Neurophysiol. 101(6):2961-2973.

Lipovsek M, Im GJ, Franchini LF, Pisciottano F, Katz E, Fuchs PA, Elgoyhen AB. 2012. Phylogenetic differences in calcium permeability of the auditory hair cell cholinergic nicotinic receptor. Proc Natl Acad Sci U S A. 109(11):4308-4313.

Liu H, Pecka JL, Zhang Q, Soukup GA, Beisel KW, He DZ. 2014. Characterization of transcriptomes of cochlear inner and outer hair cells. J Neurosci. 34(33):11085-11095.

Lynch $M$, Force A. 2000. The probability of duplicate gene preservation by subfunctionalization. Genetics 154(1):459-473.

Manley GA. 2000. Cochlear mechanisms from a phylogenetic viewpoint Proc Natl Acad Sci U S A. 97(22):11736-11743.

Manley GA. 2010. The origin and evolution of high-frequency hearing in (most) mammals. Hear Res. 270(1-2):2-3.

Manley GA. 2012. Evolutionary paths to mammalian cochleae. J Assoc Res Otolaryngol. 13(6):733-743.

Manley GA, Popper AN, Fay RR. 2004. Evolution of the vertebrate auditory system. New York: Springer-Verlag.

Matsubara A, Laake JH, Davanger S, Usami S, Ottersen OP. 1996 Organization of AMPA receptor subunits at a glutamate synapse: a quantitative immunogold analysis of hair cell synapses in the rat organ of Corti. J Neurosci. 16(14):4457-4467.

Moser T, Beutner D. 2000. Kinetics of exocytosis and endocytosis at the cochlear inner hair cell afferent synapse of the mouse. Proc Natl Acad Sci U S A. 97(2):883-888.

Mostafapour SP, Cochran SL, Del Puerto NM, Rubel EW. 2000. Patterns of cell death in mouse anteroventral cochlear nucleus neurons after unilateral cochlea removal. J Comp Neurol. 426(4):561-571.

Murrow BW, Fuchs PA. 1990. Preferential expression of transient potassium current (IA) by 'short' hair cells of the chick's cochlea. Proc Biol Sci. 242(1305):189-195.

Okoruwa OE, Weston MD, Sanjeevi DC, Millemon AR, Fritzsch B, Hallworth R, Beisel KW. 2008. Evolutionary insights into the unique electromotility motor of mammalian outer hair cells. Evol Dev. 10(3):300-315.

Peter IS, Davidson EH. 2011. Evolution of gene regulatory networks controlling body plan development. Cell 144(6):970-985.

Platzer J, Engel J, Schrott-Fischer A, Stephan K, Bova S, Chen H, Zheng H, Striessnig J. 2000. Congenital deafness and sinoatrial node dysfunction in mice lacking class D L-type $\mathrm{Ca}^{2+}$ channels. Cell 102(1):89-97.

Roberts WM, Jacobs RA, Hudspeth AJ. 1990. Colocalization of ion channels involved in frequency selectivity and synaptic transmission at presynaptic active zones of hair cells. J Neurosci. 10(11):3664-3684.

Scheffer DI, Shen J, Corey DP, Chen ZY. 2015. Gene expression by mouse inner ear hair cells during development. J Neurosci. 35(16):6366-6380. 
Schultz JA, Zeller U, Luo ZX. 2017. Inner ear labyrinth anatomy of monotremes and implications for mammalian inner ear evolution. J Morphol. 278(2):236-263.

Shera CA, Guinan JJ Jr 1999. Evoked otoacoustic emissions arise by two fundamentally different mechanisms: a taxonomy for mammalian OAEs. J Acoust Soc Am. 105(2):782-798.

Slepecky NB. 1996. Structure of the mammalian cochlea. New York: Springer.

Son EJ, Wu L, Yoon H, Kim S, Choi JY, Bok J. 2012. Developmental gene expression profiling along the tonotopic axis of the mouse cochlea. PLoS One 7:14.

Spassova M, Eisen MD, Saunders JC, Parsons TD. 2001. Chick cochlear hair cell exocytosis mediated by dihydropyridine-sensitive calcium channels. J Physiol (Lond). 535(Pt 3):689-696.

Stapelbroek JM, Peters TA, van Beurden DH, Curfs JH, Joosten A, Beynon $A J$, van Leeuwen $B M$, van der Velden $L M$, Bull $L$, Oude Elferink RP, et al. 2009. ATP8B1 is essential for maintaining normal hearing. Proc Natl Acad Sci U S A. 106(24):9709-9714.

Tamura K, Peterson D, Peterson N, Stecher G, Nei M, Kumar S. 2011. MEGA5: molecular evolutionary genetics analysis using maximum likelihood, evolutionary distance, and maximum parsimony methods. Mol Biol Evol. 28(10):2731-2739.

Tierney TS, Russell FA, Moore DR. 1997. Susceptibility of developing cochlear nucleus neurons to deafferentation-induced death abruptly ends just before the onset of hearing. J Comp Neurol. 378(2):295-306.

Wang $H$, Yang H, Shivalila CS, Dawlaty MM, Cheng AW, Zhang F, Jaenisch R. 2013. One-step generation of mice carrying mutations in multiple genes by CRISPR/Cas-mediated genome engineering. Cell 153(4):910-918.

Yang Z. 2007. PAML 4: phylogenetic analysis by maximum likelihood. Mol Biol Evol. 24(8):1586-1591.

Zhang J, Nielsen R, Yang Z. 2005. Evaluation of an improved branch-site likelihood method for detecting positive selection at the molecular level. Mol Biol Evol. 22(12):2472-2479.

Zheng J, Shen W, He DZ, Long KB, Madison LD, Dallos P. 2000. Prestin is the motor protein of cochlear outer hair cells. Nature 405(6783):149-155. 